\title{
Vers un Calcul des Significations dans l'Analyse des Langues
}

[Para um Cálculo de Significados na Análise da Linguagem]

\author{
Jean-Pierre Desclés®
}

\begin{abstract}
Résumé: L'article présente quelques réflexions épistémologiques sur le programme de recherche dont l'objet est l'étude de l'activité de langage exprimée par les langues, activité qui ne se réduit pas à la simple communication et à l'expression de la pensée. Il présente certains concepts importants de la théorie de l'énonciation en les articulant avec des schèmes sémantico-cognitifs qui représentent les significations d'unités grammaticales et lexicales et qui sont engendrés dans le formalisme de la Logique Combinatoire de Curry par des compositions et des transformations d'opérateurs primitifs ancrés sur les activités cognitives de perception et d'action mais non réduits à ces activités.
\end{abstract}

Mots clés: Activité de langage. Sémantique. Énonciation. Schème cognitifs. Logique combinatoire de Curry.

Resumo: O artigo apresenta algumas reflexões epistemológicas sobre o programa de pesquisa, cujo objeto é o estudo da atividade da linguagem expressa pelas línguas, atividade que não se reduz à simples comunicação e à expressão do pensamento. Ele apresenta certos conceitos importantes da teoria da enunciação articulando-os com esquemas semântico-cognitivos que representam os significados de unidades gramaticais e lexicais e que são gerados no formalismo da Lógica Combinatória de Curry por composições e transformações de operadores primitivos ancorados em atividades cognitivas de percepção e ação, mas não reduzidos a essas atividades.

Palavras-chave: Atividade da linguagem. Semântica. Enunciação. Esquemas cognitivos. Lógica combinatória de Curry.

L'étude formelle de la syntaxe des langues a été poussée très loin avec la Grammaire Générative, avant d'aboutir au "modèle minimaliste " actuel de N. Chomsky, tandis que R. Montague et d'autres logiciens développaient une sémantique formelle et intension- nelle en prenant appui sur la syntaxe formalisée par les Grammaires Catégorielles héritées de l'Ecole polonaise (S. Lesniewski et K. Ajdukiewicz). Parallèlement, la linguistique contemporaine a vu émerger deux autres courants théoriques importants: d'un côté, la

\footnotetext{
${ }^{*}$ Pr. émérite, Sorbonne Université. Docteur d'état en Mathématiques, Université de Paris - René Descartes. Docteur Honoris causa de l’Université de Sofia (Bulgarie). Membre de l'Académie Internationale de Philosophie des Sciences. E-mail: jeanpierre.descles@gmail.com.
} 
théorie de l'énonciation, initialisée par les travaux de Ch. Bally et d'É. Benveniste, puis développée sous une forme plus formelle par A. Culioli et ses disciples et, d'un autre côté, le courant de la sémantique cognitive, qualifiée comme telle aux USA mais déjà abordée en Europe sous d'autres étiquettes. Analysant la distinction entre "syntaxe, sémantique, pragmatique » de Ch. Morris, l'épistémologue G.-G. Granger a mis en évidence l'importance de la pragmatique dans l'analyse des langues et dans la construction de significations préalables aux descriptions dénotatives, ramenées, à la suite d'A. Tarski, à une construction des valeurs de vérité dans des modèles ensemblistes.

"La notion de conditions pragmatiques de la communication linguistique est demeurée, depuis Morris, très ambiguë. Carnap envisage la pragmatique comme une étude essentiellement empirique, dont il considère du reste qu'elle forme "la base " de la linguistique.»

"Bar-Hillel et Montague proposent de réduire l'aspect pragmatique de l'étude de la langue à l'examen des "indexical expressions » de Peirce, c'est-à-dire, selon ces auteurs, des expressions " dont la référence ne peut être déterminée sans la connaissance de leurs contextes d'usage. (Montague)»
(G.-G. Granger, Langages et épistémologie, Paris, Klincksieck, 1979: 165).

Dans l'étude de la sémantique des langages de la logique moderne et des langages de programmation, la pragmatique et la signification ne semblent pas opératoires, ce qui n'est évidemment pas le cas lorsqu'on étudie les langues naturelles. Pour définir le rôle des opérations de "prise en charge " d'un contenu prédicatif, la théorie de l'énonciation introduit explicitement des représentations pragmatiques dans les descriptions métalinguistiques qu'elle élabore, en distinguant toutefois et implicitement une pragmatique interne (et formelle) d'une pragmatique externe, ce qui revient à ne pas assimiler d'un côté, les rôles abstraits d'énonciateur et de coénonciateur, insérés dans un référentiel énonciatif autonome et détaché du monde externe, avec, d'un autre côté, les locuteurs et allocutaires empiriques plongés dans un monde spatio-temporel directement accessible à sa perception et aux actions qui s'y exercent. L'analyse de la signification d'un texte par des représentations pragmatiques internes où s'inscrivent formellement les relations entre énonciateur et coénonciateur, n'épuise pas le sens d'un discours, en particulier d'un texte, car la caractérisation profonde du sens nécessite plusieurs connaissances pragmatiques externes relatives aux locuteurs et al- 
locutaires, à leurs intentions respectives, que les discours ne révèlent pas toujours, et à leurs environnements extralinguistiques.

Dans notre approche formelle d'une sémantique cognitive et énonciative, la construction de la signification d'un discours, doit être première et précéder l'étude (beaucoup plus complexe) de son sens. Cette construction fait appel non seulement à des outils logiques mais également à des conceptualisations topologiques, ce qui rejoint G.G. Granger, qui a su reconnaître, dans La Pensée de l'espace, une articulation continuelle entre des interprétations géométriques et des calculs logicoalgébriques.

Le présent article expose quelques brèves réflexions épistémologiques sur ce qui nous semble être le programme essentiel de recherche de la linguistique théorique et générale: caractériser la complexité cognitive de l'activité de langage que les langues manifestent par des systèmes sémiotiques variés (étudiés par la typologie des langues), en ne réduisant l'activité de langage à une simple communication et à l'expression de la pensée. Nous évoquerons des exemples élémentaires de constructions de significations, aussi bien grammaticales que lexicales, en utilisant une logique d'opérateurs quelconques.

\section{Positions épistémologiques}

Commençons par énumérer quelques prises de position épistémologique.

P1: Les langues sont des systèmes sémiotiques composés de lexèmes, de grammèmes et d'unités discursives qui organisent les énoncés et leurs enchaînements dans des discours.

P2: Les systèmes sémiotiques des langues ouvrent à leurs utilisateurs la capacité de construire des dialogues. La fonction du langage humain est avant tout dialogique, l'expression de la pensée et la communication sont dérivées de cette fonction beaucoup plus fondamentale. A propos du «dialogue », F. Jullien précise:

"Entendons donc plus complètement ce dia-du dialogue: il ne désigne pas seulement l' "entre» de la parole (entre l'interlocuteur et moi) mais aussi l'" à travers" et le déroulement (dia comme dia-doxazein, cf. Philèbe, 38, c); c'est au travers même $d u$ logos, de son extension comme de son épaisseur, mais devenant de part en part translucides, que je m'entends avec l'interlocuteur et chemine avec lui.»

"Car seule cette communauté de l'entente, et par suite de l'intelligible, fait et garantit la validité de la pensée. » 
(F. Jullien, Si parler va sans dire, Paris, Seuil, 2006, p. 97)

Cette communauté de l'entente n'est pas sans évoquer le fameux dialogue du De Magistro entre Saint Augustin et son fils Adeodat. Il faut cependant reconnaître que "l'entente » n'est pas toujours pleinement réalisée par l'échange dialogique, ce qu'A. Culioli, en reprenant un constat du sociologue $P$. Bourdieu, se plaît souvent à souligner: "La compréhension est un cas particulier du malentend $u » 11$

P3: La construction des significations précède les dénotations. Une telle proposition soulève plusieurs questions posées aux linguistes. Comment construire les significations à partir d'une analyse des expressions linguistiques? Faut-il faire appel à plusieurs niveaux de représentations, selon une approche polystratale ou bien peut-on se contenter d'une simple relation (qualifiée alors d'arbitraire) entre un signifiant et son signifié?

Lorsqu'on cherche à relier des niveaux d'analyse, on doit s'interroger sur les formalismes qui sont susceptibles de prendre adéquatement en charge les différentes formes de représentations et les opérations qui les constituent et opèrent sur elles pour les transformer. Vouloir dépasser l'étude de la seule syntaxe des langues, c'est faire appel à des concepts qui ne sont plus uniquement classificatoires (donc plus complexes que les structures arborescentes d'une forme particulière de syntaxe), et rechercher des formalismes logico-algébriques et géométriques plus adéquats pour représenter les relations entre syntaxe et sémantique, en sachant en exploiter les propriétés mathématiques utiles.

Si la linguistique, en tant que science humaine, cherche à faire complètement partie de la famille des sciences dites "dures », ne doit-elle pas chercher à mathématiser les concepts de façon à « expliquer » le fonctionnement de l'activité de langage? Des réponses sont parfois avancées aussi bien par des philosophes que par des linguistes:

"Il semble qu'on ne puisse parvenir à concevoir et à organiser de façon véritablement systématique le langage que si l'on se fonde sur la systématique propre des Mathématiques et que l'on emprunte ses critères. »

(E. Cassirer, La philosophie des formes symboliques. 1. le langage, Paris, Editions de Minuit, 1953/1972, p. 73)

"Disons que le linguiste aura parfois des concepts-clés à portée de main (par ex. application, struc-

\footnotetext{
${ }^{1}$ Voir A. Culioli, Variations sur la linguistique, Entretiens avec Frédéric Fau, Paris, Klincksieck, 2002, p. 28.
} 
ture, ordre), parfois l'élaboration sera lente (ainsi en est-il de l'utilisation de la topologie en linguistique et de la logique combinatoire), parfois il faudra tout faire (ainsi dans le domaine des modalités). »

(A. Culioli, «La formalisation en linguistique ", Cahiers pour l'analyse, Vol. 9, 1968)

La linguistique emprunterait ainsi un chemin analogue à celui qu'a suivi Galilée, lorsqu'il a su quitter la seule métaphysique aristotélicienne pour une véritable mathématisation des concepts impliqués dans l'explication de la chute des corps.

P4: La linguistique ne doit pas se satisfaire d'approches théoriques qui semblent vouloir rester étrangères les unes aux autres et s'enfermer dans des " écoles » aux frontières bien tracées et peu étanches. C'est actuellement le cas avec d'un côté, une "sémantique formelle » qui s'appuie, sur la logique et sur des analyses sémantiques des langues analogues à celles données par R. Montague, et d'un autre côté, une " sémantique cognitive ", développée entre autres, aux USA par G. Lakoff, C.J. Fillmore, G. Lakoff, R. Langacker, L. Talmy ... et, en Europe, par G. Guil- laume, B. Pottier, R. Thom, H. Seiler, W. Wildgen.

Remarque: Une rencontre entre spécialistes de la sémantique cognitive européens (A. Culioli, J.-P. Desclés, D. Dubois, B. Pottier, H. Seiler, W. Wildgen) et nord-américains (R. Langacker, L. Talmy) a eu lieu à Sion (Suisse), du 6 au 10 septembre 1993 3 , elle a permis de comparer les convergences et divergences.

Pour la linguistique, il s'agit de répondre à des questions comme:

- Quelle est l'interaction entre l'activité de langage et les activités cognitives (plus ou moins intentionnelles) de perception et d'action?

- Comment articuler les images, les représentations iconiques et figuratives avec des formulations sémantiques plus symboliques?

- Comment les représentations symboliques sont-elles compatibles avec des calculs et des déductions formelles chargées de construire les interprétations des unités linguistiques agencées dans les énoncés d'une langue et, plus généralement, de construire les interprétations globales des discours et des textes?

P5: Il devient indispensable d'articuler les deux courants, celui de la sé-

\footnotetext{
${ }^{2}$ Cet article a été repris dans A. Culioli, Pour une théorie de l'énonciation, formalisme et opération de repérage, Tome 2, Paris, Ophrys, 1999 , p. 25.

${ }^{3}$ G. Lüdi et Cl. Zuber (eds), Linguistique et modèles cognitifs. Contributions à l'Ecole d'été de la Société Suisse de Linguistique, ARBA 3, Acta Romanica Basiliensia. XXX, Université de Bâle et Institut Universitaire Kurt Bösch, 1995.
} 
mantique formelle et celui de la sémantique cognitive, avec la théorie de l'énonciation (au sens restreint de la théorie des opérations énonciatives, prédicatives et de détermination), développée principalement en Europe, à partir des travaux philosophiques et linguistiques de E. Pauliny, M. Buber, K. Bühler, Ch. Bally, É. Benveniste, A. Culioli ..., et pour être en mesure de répondre aux questions suivantes:

- Comment tenir compte des expressions indexicales (et expressions apparentées) qui manifestent dans les discours, les rôles de l'énonciateur et du coénonciateur?

- Comment représenter, par des opérations formalisées, les prises en charge d'un contenu prédicatif effectuées par l'énonciateur qui souhaite indiquer « l'attitude » qu'il adopte par rapport au contenu qu'il transmet au coénonciateur?

P6: Le traitement de masses de données à l'aide d'ordinateurs de plus en plus rapides et toutes les réflexions menées par l'informatique théorique ont débouché sur un ensemble de concepts opératoires puissants. C'est le cas, par exemple, des grammaires et langages formels et de la théorie des automates (avec, par exemple, les travaux de N. Chomsky et de M. Schützenberger); c'est également le cas de la prise en compte des différents types de données et des différents types d'opérateurs (avec les types fonctionnels de Church). Les sciences humaines, et en particulier la linguistique, ne devraient-elles pas faire plus systématiquement appel à ces concepts opératoires et aux traitements qu'ils permettent, afin de pouvoir rectifier, modifier, faire varier les données sur lesquelles elles travaillent, en cherchant aussi à valider, par des simulations expérimentales, les constructions théoriques et les représentations formelles que ces disciplines ont élaborées? Lorsqu'elles sont menées avec rigueur et dans un souci de généralisation, les simulations de ce genre, permettent d'envisager parfois et ultérieurement diverses applications solides et complexifiables, car justifiées par des réflexions théoriques préalables qui font mieux comprendre la nature et la limite des représentations utilisées.

P7: L'étude de l'activité de langage est devenue interdisciplinaire. Cependant, si l'on veut bénéficier d'une " bonne interdisciplinarité », quelques principes épistémologiques doivent être respectés Mentionnons en deux.

Premier principe. Il s'agit de savoir " écouter " les autres disciplines afin de se donner les moyens de complexifier là où cela devient nécessaire. Ce n'est peut-être pas le cas de ce qui peut être observé dans le déroulement de certaines recherches de neuroscience computationnelle portant sur le langage car, bien qu'elles annoncent vouloir " expliquer » le fonctionnement du langage, 
ces recherches opèrent souvent uniquement à partir des seuls mots lexicaux, alors que, depuis longtemps, la linguistique a montré que «le mot " n'est pas une unité très opératoire, car déjà très construite, les "bonnes » unités de base étant plutôt les grammèmes (unités grammaticales en nombre fini dans une langue) et les lexèmes.

Second principe. Il s'agit de se méfier d'une simulation trop rapide dans laquelle l'utilisation d'algorithmes n'est pas préalablement justifiée par des conceptions théoriques, préalablement discutées et évaluées. C'est peut-être l'interrogation que l'on peut se poser à propos d'un usage actuel de l'Intelligence Artificielle (IA), qui fonde uniquement des décisions sur des algorithmes résultant de processus d'apprentissage (parfois profond) d'informations extraites, par de traitements statistiques, de vastes corpus, préalablement annotés ou non. Or, certains bons spécialistes mettent en garde sur l'utilisation contrôlée et justifiée des statistiques: "Laissez-moi maître du choix des données et je vous prouve ce que je veux. » On peut aussi réfléchir à ce que disait, avec l'ironie qu'on lui connaît, Winston Churchill: «Je ne crois aux statistiques que lorsque je les ai moimême falsifiées ». Les statistiques sont évidemment très utiles dans les sciences humaines et en linguistique mais elles n'épargnent pas leurs utilisateurs de mener une sérieuse réflexion sur la nature, le choix et le traitement des données que l'on utilise dans les apprentissages et sur leur adéquation à répondre à des problèmes qui sont, par ailleurs, bien posés et pertinents.

\section{Théorie de l'énonciation}

Nous allons maintenant rappeler quelques éléments de l'énonciation qui joue un rôle important dans l'activité de langage. $4^{4}$ Durant l'époque médiévale, certaines logiciens avaient déjà fait émerger quelques concepts énonciatvistes sans qu'il y ait eu cependant une suite jusqu'au $\mathrm{XX}^{\mathrm{e}}$ siècle, là où un philosophe comme M. Buber (1923) s'interroge sur le statut très particulier des personnes je et $t u$ :
"Les bases du langage ne sont pas des mots isolés, ce sont des couples de mots. (...) L'une des bases $d u$ langage, c'est le couple Je-Tu. (...) L'autre est le couple Je-Cela, dans lequel on peut rem- placer Cela par Il ou Elle sans que le sens en soit modifié. (...) Donc le Je de l'homme est double, lui aussi. (...) Car le Je du couple ver- bal Je-Tu est autre que celui $d u$

\footnotetext{
${ }^{4}$ M. Colas-Blaise, L. Perrin et G. M. Tore, L'énonciation aujourd'hui, un concept clé des sciences du langage, Paris, Lambert-Lucas, 2016.

${ }^{5}$ M. Buber. Je et Tu, [Ich und Du, 1923], Paris, Aubier-Montaigne, 1969.
} 
couple verbal Je-Cela.»

(M. Buber, Je et Tu, 1923/1969, p. 19)

À Genève, Ch. Bally (1932) ${ }^{6}$ décompose la phrase en un modus et un dictum, le modus prenant toute sa pertinence dans une théorie de l'énonciation. En France, E. Benveniste $(1965,1974) 7$ écrit, sans aucune référence aux auteurs précédents, des articles fondamentaux sur la subjectivité dans le langage, sur le jeu de je et tu opposés à il (qualifié comme une "non personne ») et il distingue les deux «plans » d'énonciation, le « discours » et " histoire ». A. Culioli $(1973,1999){ }^{8}$ reprend un peu plus formellement ce jeu entre les marqueurs personnels $j e$ et $t u$, avec différentes formes de repérage dans les énonciations directes et indirectes, en insistant, dans la construction d'un énoncé, sur les rôles d'énonciateur et de coénonciateur; il entreprend également une approche énonciative des opérations aspectuelles, en dégageant le rôle particulier qu'occupe la situation d'énonciation dans la construction de chaque énoncé.

En prenant explicitement appui sur les différentes conceptualisations précé- dentes, j'ai entrepris, depuis 1976 de chercher à formaliser systématiquement les opérations d'énonciation composées avec les opérations de prédication et de détermination, en les intégrant dans des représentations métalinguistiques (qui décrivent les expressions linguistiques); pour cela, j'utilise des « langages applicatifs » dans lesquels différents types d'opérateurs s'appliquent à des opérandes afin de construire des propositions, des énoncés, et plus généralement, des discours.

La prise en compte de l'énonciation a conduit à distinguer deux pragmatiques, l'une interne et l'autre externe. La pragmatique interne est spécifique à la «Langue » (au sens de Saussure); cette pragmatique interne est, nous semble-t-il, l'une des caractéristiques les plus fondamentales du langage des humains puisque les utilisateurs (énonciateurs et coénonciateurs) se représentent leurs relations à l'intérieur du système sémiotique (une langue) qu'ils utilisent. Les langues manifestent la présence de signes indexicaux (par exemple je et $t u$, ici et là-bas, aujourd'hui et hier), qui sont justement les traces des rôles d'énonciateur et de coénonciateur et de leurs relations dans une énonciation. Ces rôles, désignés respectivement par EGO et par TU, sont localisés dans

\footnotetext{
${ }^{6}$ Ch. Bally. Linguistique générale et linguistique française, Berne, Editions Francke, 1934/1965;

${ }^{7}$ E. Benveniste, Problèmes de linguistique générale, Paris, Gallimard, 1965, 1974).

${ }^{8}$ A. Culioli, Pour une linguistique de l'énonciation, Tomes 1,2,3, Paris, Ophrys, 1990, 1999.

${ }^{9}$ J.-P. Desclés, «Quelques opérations énonciatives » in J. David et R. Martin, Logique et niveaux d'analyse linguistique, Paris, Klincksieck, 1976; repris in J.-P. Desclés et G. Guibert, Le dialogue, fonction première du langage, Analyse énonciative de textes, Paris, Honoré Champion, 2011, pp. 49-73.
} 
le référentiel énonciatif commun 'REN', que construit l'acte d'énonciation, en le détachant du référentiel externe 'REX': " énoncer, c'est construire un référentiel dialogique autonome $\gg 10$, donc indépendant du monde externe où se situent physiquement le locuteur et ses auditeurs empiriques. Les rôles de EGO et TU ne doivent pas être confondus avec ceux tenus par les locuteurs et les allocutaires, ces derniers sont des êtres déterminés et localisés dans un monde externe, celui d'une pragmatique externe, avec laquelle on pourra tenter d'appréhender «le sens » d'un énoncé où interviennent le contexte de l'environnement externe d'un locuteur déterminé et de son (ou de ses) allocutaire(s) et de leurs intentions éventuelles. Dans certains cas, comme dans le soliloque, EGO et TU peuvent être instanciés par un même locuteur externe, qui alors assume, en même temps, deux rôles, celui de EGO et celui de TU: "Penser c'est déjà dialoguer avec soi, vieux truisme des Grecs (Thétète, 189, c) », comme nous le rappelle F. Jullien.

A partir d'analyses sémantiques du verbe «être » et de ses équivalents dans des langues non indo-européennes, A. Culioli détermine un relateur (ou opérateur binaire) abstrait qui a un rôle structurant dans l'analyse de l'activité de langage en reliant une entité repérée (avec une valeur référentielle moins déterminée) à une entité qui lui sert de repère (mieux déterminé); ce relateur de repérage est générique au sens suivant: selon les énoncés où il présente une occurrence, il acquiert une signification (ou valeur) plus précise, soit une identification' $(=)$, soit une forme de différenciation $(\neq)$ avec des propriétés nettement différentes. Les identifications et différenciations prennent, selon leurs contextes linguistiques, des valeurs encore plus précises: l'équivalence et l'identité spécifient l'identification (=); les spécifications de la différenciation $(\neq)$ sont l'appartenance $(\in)$ d'un élément à une classe ou l'inclusion $(\subseteq)$ entre classes ou la relation de localisation d'un objet dans un lieu ou d'un lieu par rapport à un autre lieu ou encore la relation méréologique $(\varepsilon)$ d'une partie à un tout. Les énoncés suivants exemplifient ces différentes formes de repérage:

Brasilia est $(=)$ la capitale du Brésil. Macapa est $(\in)$ une ville du Brésil. Les villes sont $(\subseteq)$ très peuplées au Brésil. Macapa est en (localisation) Amazonie. La Tour Eiffel est (ع) une image de Paris.

Pour exprimer ces différentes valeurs sémantiques du repérage, indiqué au moyen du verbe nettement polysémique est du français ou d'une forme équivalente dans diverses langues indo-européennes, la plupart

\footnotetext{
10 «Nous rappelons qu'énoncer, c'est construire un espace, orienter, déterminer, établir un réseau de valeurs référentieles, bref un système de repérage » A. Culioli, Pour une linguistique de l'énonciation, Tome 2, 1973/1999, o.c., p. 49.
} 
des langues non-indoeuropéennes emploient des lexèmes différents; par exemple, en chinois, les trois marqueurs shi, zai et you traduisent, selon les contextes, les valeurs de être et de avoir du français. ${ }^{11}$

Aux valeurs ' $=$ ' et ' $\neq$ ' du repérage abstrait, il convient d'ajouter la valeur de " mise en rupture », ou simplement de rupture (\#), qui est une forme de "négation forte ", distincte de la négation faible indiquée par la seule différenciation $(\neq)$; on peut ainsi comparer les deux négations exprimées par les énoncés:

\section{Cayenne n'est pas (\#) au Brésil}

Rio n'est plus $(\neq)$ la capitale du Brésil.

Les trois valeurs abstraites, avec leurs propriétés mathématiques qui les différencient, constituent le système de repérage $\{=, \neq, \#\}$ avec le relateur générique, noté ' $\epsilon$ ' par Culioli pour rappeler les différentes valeurs qu'il est susceptible de prendre en contexte. Ce repérage par ' $\in$ ' exprime des valeurs sémantique encore plus spécifiques selon différents domaines 12 .

- Le domaine des rôles énonciatifs:

EGO / TU // IL avec:

TU se différencie de EGO:

[TU $\neq \mathrm{EGO}$ ] tandis que IL, « l'absent du dialogue », est en relation de rupture avec à la fois EGO et TU:

[IL \# EGO] \& [IL \# TU]

- Le domaine des lieux déictiques spatiaux:

ICI / LÀ-BAS // AIILLEURS avec:

pour chaque $x \in I C I$ :

$[\mathrm{y} \in \mathrm{LÀ}-\mathrm{BAS} \quad \Rightarrow \mathrm{y} \neq \mathrm{x}]$;

pour chaque $\mathrm{y} \in \mathrm{LÀ}-\mathrm{BAS} \cup \mathrm{ICI}$ :

$[\mathrm{z} \in$ AILLEURS $\Rightarrow \mathrm{z} \# \mathrm{y}$ ]

- Le domaine des lieux temporels, AUJOURD'HUI / HIER ou DEMAIN // UN JOUR avec:

pour chaque t AUJOURD'HUI:

$\left[\mathrm{t}^{\prime} \in \mathrm{HIER} \quad=>\mathrm{t}^{\prime} \neq \mathrm{t}\right]$

pour chaque $t^{\prime}$ AUJOURD'HUI $U$ HIER:

$\left[\mathrm{t}^{\prime \prime} \in \mathrm{UN}-\mathrm{JOUR}=>\mathrm{t}^{\prime \prime} \# \mathrm{t}^{\prime}\right]$

Le concept de changement de référentiel est, selon nous, l'un des plus importants de la théorie de l'énonciation, du moins telle que nous la défendons, car ce concept permet d'expliquer une propriété de l'activité de langage manifestée dans l'analyse sémantique de nombreux marqueurs grammaticaux et dans l'organisation discursive ellemême. 13 Comme nous l'avons déjà dit, lorsque l'énonciateur EGO prend la parole, il construit un référentiel énonciatif REN commun avec son coénonciateur $\mathrm{TU}$, en se déconnectant du référentiel externe REX, ce qui revient à établir une rupture (\#) entre leurs

\footnotetext{
${ }^{11}$ Pour répondre à une demande de l'UNESCO, A. Culioli et J.-P. Desclés, avec la collaboration de R. Kaboré et Dj. Kouloughli, ont remis en 1980, le rapport Systèmes de représentations linguistiques et métalinguistiques. Les catégories grammaticales et le problème de langues peu étudiées, 141 pages, publié dans la Collection ERA 642, Université Paris 7, 1981.

12 Voir J.-P. Desclés et G. Guibert, 2011, o.c. , pp. 15-121.

${ }^{13}$ Les changements de référentiels permettent de formaliser les oppositions entre " premier et deuxième plans " rencontrés dans l'analyse textuelle (par exemple chez B. Combettes), la polyphonie et les «points de vue » qu'Alain Rabatel développe dans La construction textuelle du point de vue, Paris, Delachaux et Niestlé, 1998.
} 
éléments constitutifs respectifs: [REN \# REX], tout en se donnant la possibilité d'établir certaines identifications, par exemple par des synchronisations locales exprimées par l'emploi du "présent historique », le "présent de reportage », " imparfait de politesse » pour ne prendre que ces exemples. ${ }^{14}$ L'activité de langage donne la possibilité de procéder à d'autres changements de référentiels, par exemple, en construisant un référentiel narratif RNA, où les situations d'une narration sont repérées entre elles (par indentification ou par différenciation) mais sont en rupture avec les situations du référentiel énonciatif REN: [RNA \# REN] (exemple: Un jour, le chaman est devenu un jaguar; le lendemain, il est redevenu un homme). Le langage humain donne à ses utilisateurs la capacité de reprendre, plus ou moins directement et fidèlement, les propos d'un tiers, en créant un référentiel des discours rapportés (REN2), les autres espèces animales n'ayant pas, nous semble-t-il, cette capacité cognitive et langagière. L'énonciateur a également la possibilité d'énoncer une hypothèse en localisant cette situation, qui est simplement envisagée comme possible, dans le référentiel des situations hypothétiques (RHYP), pour en tirer des conséquences et les comparer éventuellement avec les situations actualisées dans son référentiel REN (ex.: Si tu es arrivé, je te rapporte aussitôt les objets que tu m'as prêtés / Si tu fais un pas de plus, je tire / Si 27 n'a aucun diviseur entier, il faut l'ajouter aux nombres premiers). Dans une énonciation contrefactuelle, il s'agit d'une hypothèse énoncée par l'énonciateur et présentée comme étant en complète contradiction avec ce qui est connu et actualisé dans le référentiel énonciatif REN ou dans le référentiel externe REX ou encore dans un référentiel narratif RNA déjà construit (ex.: $S i$ son père avait été présent ce jour-là, sa fille ne serait pas partie, seule, en Amazonie). Les constructions discursives mettent souvent en jeu, au cours de leurs énonciations, plusieurs changements de référentiels; par exemple, au cours d'une narration, l'énonciateur peut évoquer des savoirs et connaissances partagées, citer des proverbes ou des situations relatives à des narrations mythiques. L'énonciation "médiative » (dite aussi " évidentielle ») est la production discursive d'une hypothèse simplement plausible (à ne pas confondre avec l'énonciation d'un jugement portant sur la probabilité d'une situation) qui impose, pour son explication lorsqu'elle est réclamée, une articulation entre deux référentiels. Pour illustrer ce genre d'énonciation médiative, imaginons quelqu'un qui, ayant ouvert les fenêtres, constate que «la

\footnotetext{
14 J.-P. Desclés et Z. Guentchéva, "Référentiels aspectuo-temporels: une approche formelle et cognitive appliqué au français ", Bulletin de la Société de Linguistique de Paris, 56 (1), 2012, pp. 95-127.
} 
chaussée est mouillée » (q) et juge que ce constat est pour lui un indice en faveur de l'hypothèse plausible « il a plu cette nuit »(p), sachant que l'on a l'implication "s'il pleut alors la chaussée est mouillée » $(\mathrm{p}=>\mathrm{q})$; dans ce cas, il énonce alors directement la plausibilité de p: Il a donc plus cette nuit ou encore Ainsi, il aurait plu cette nuit; pour produire cette énonciation, il a effectué une inférence par abduction (au sens de C. S. Peirce), c'est-à-dire: (i) ayant constaté q, (ii) il imagine une hypothèse $p$, ayant la connaissance de (iii) l'implication $[\mathrm{p}=>\mathrm{q}]$, ce qui l'amène finalement à pouvoir (iv) énoncer $p$, en signalant toutefois à son coénonciateur, par la forme linguistique retenue, que $\mathrm{p}$ est pour lui, énonciateur, une hypothèse seulement plausible pas nécessairement vraie; par cette énonciation médiative, l'énonciateur dégage sa responsabilité, ce qui n'aurait pas été le cas s'il avait affirmé: Il a plu cette nuit. En présentant un fait comme plausible, l'énonciateur fait savoir qu'il existe éventuellement d'autres hypothèses plausibles susceptibles d'avoir entraîné l'actualisation du fait constaté (ex.: « l'arroseuse municipale est déjà passée dans la rue »).

Dans la décomposition d'une "phrase », par Ch. Bally ${ }^{15}$, en un modus et un dictum, ce dernier est analogue à ce que A. Culioli, à la suite des réflexions menées à propos du $\lambda \varepsilon \varkappa \tau$ tov de la philosophie stoïcienne, appelle « lexis », c'est-à-dire une expression propositionnelle structurée qui est seulement dicible car pas encore dite et, pour être dite, elle doit obligatoirement être prise en charge par un énonciateur et être ainsi énoncée selon diverses modalités exprimées par le modus. C'est le modus qui indique l'attitude que l'énonciateur adopte par rapport au contenu (la lexis ou le dictum) exprimé par l'énoncé, et non pas par rapport à l'énoncé luimême.

Remarque: De nombreuses approches énonciativistes caractérisent la prise en charge comme « l'attitude de l'énonciateur vis-à-vis de son énoncé », ce qui nous paraît être un contresens puisque l'énoncé est justement le résultat et par conséquent la manifestation de cette attitude.

Dans la formalisation des opérations énonciatives, le modus prend la forme d'un opérateur complexe qui s'applique à son opérande (la lexis ou le dictum), construit par des opérations prédicatives et déterminatives, pour finalement construire un énoncé, manifesté par une forme signifiante exprimée dans le système sémiotique d'une langue. En désignant par @ l'opération d'application d'un opérateur à son opérande, l'énoncé est ainsi décomposé et représenté par l'expression applicative 'modus@dictum', notée plus simplement 'modus (dictum)'.

\footnotetext{
15 Ch. Bally, 1934/1965, o.c., pp. 35-52. Le dictum est un contenu organisé sous une forme propositionnelle, mise sous la dépendance d'un modus. Dans notre approche applicative, le modus s'applique au dictum (ou à une lexis).
} 
Le modus est un opérateur qui a pour constituant, minimal et nécessaire, l'opérateur EGO-DIT, à la base de toute énonciation. Cet opérateur se compose avec l'opérateur de " processus inaccompli », noté $\mathrm{PROC}_{\mathrm{J} 0}$, qui s'actualise sur un intervalle continu $\mathrm{J}_{0}$ d'instants, l'intervalle d'énonciation, localisé dans le référentiel énonciatif REN. L'intervalle $\mathrm{J}_{0}$ possède, à gauche, une borne fermée ' $\mathrm{g}\left(\mathrm{J}_{0}\right)$ ' indiquant un début, et, à droite, une borne ouverte ' $\mathrm{d}\left(\mathrm{J}_{0}\right)$ ' qui indique l'inaccomplissement de l'énonciation en cours à l'instant $\mathrm{T}^{0}$, d'où $\left[\mathrm{T}^{0}==_{\text {def }} \mathrm{d}\left(\mathrm{J}_{0}\right)\right]$; cela signifie que l'instant $\mathrm{T}^{0}$ n'est pas "l'instant d'énonciation », comme cela est souvent dit dans les présentations de l'énonciation.16 L'instant $\mathrm{T}^{0}$ est une borne qui sert de repère temporel fixe dans le référentiel énonciatif REN, ce qui n'est pas le cas de sa projection ' $t_{0}$ ', dans le référentiel externe REX, puisque $t_{0}$ est un instant mobile qui avance avec le flux du temps. L'opérateur $\mathrm{ENONC}_{\mathrm{J} 0}$ («EGO est en train de dire (...) » s'actualise sur $\mathrm{J}_{0}$; il est le résultat de la composition de l'opérateur EGO-DIT avec l'opérateur $\mathrm{PROC}_{\mathrm{J} 0}$ d'actualisation aspectuelle:

\section{$\mathrm{ENONC}_{\mathrm{J} 0}={ }_{\mathrm{def}} \mathrm{PROC}_{\mathrm{J} 0} \quad$ o $\quad$ EGO-DIT}

Le modus ne se réduit pas à cet opérateur énonciatif $\mathrm{ENONC}_{\mathrm{J} 0}$. $\mathrm{La}$ prise en charge énonciative du dic- tum (ou de la lexis) consiste, pour l'énonciateur, à préciser dans quel référentiel, éventuellement distinct de son référentiel énonciatif REN, il localise la situation à laquelle la lexis renvoie, en indiquant explicitement:

- l'aspectualisation du dictum, par un opérateur aspectuel (accompli ou inaccompli), qui indique les conditions de son actualisation sur un intervalle d'instants;

- le repérage temporel de la situation dénotée par le dictum aspectualisé par rapport à d'autres situations, en particulier, dans le référentiel REN, par rapport à la situation d'énonciation, c'est-à-dire l'acte de dire et d'énoncer par EGO;

- la prise en charge modale selon que le dictum aspectualisé est: ou bien simplement déclaré, ou bien présenté comme étant asserté (avec une actualisation "vraie » ou " niée ») qui alors engage l'énonciateur, ou bien comme le résultat d'un jugement épistémique indiquant une incertitude (probable, possible, improbable, contingente) de l'actualisation de la situation dénotée, ou bien encore une plausibilité de l'actualisation de la situation dénotée, à partir d'un constat d'indices et posée comme plausible par une inférence adductive;

\footnotetext{
${ }^{16}$ Notre analyse du processus n'est pas étrangère aux conceptions philosophiques sur la temporalité de A. Whitehaed, Process and Reality, New York, Macmillan, 1929.
} 
- d'autres modalités appréciatives (appréciation, désir, promesse ...) et des modalités inter-sujets (interrogation, ordre, obligation, autoobligation...).

Toutes ces modalités énonciatives se composent entre elles et avec l'opérateur $\mathrm{ENONC}_{\mathrm{J} 0}$, pour constituer l'opérateur complexe du modus. ${ }^{17}$ Prenons, par exemple, le schéma prédicatif ' $\mathrm{P}_{2} \mathrm{~T}^{2} \mathrm{~T}^{1}$ constituant un dictum; par 'MOD(-alité)' nous désignons le résultat d'une composition de différentes modalités énonciatives; ces dernières se composent avec, d'un côté, l'opérateur aspectuel $\mathrm{ASP}_{\mathrm{I} 1}$ qui actualise ${ }^{\prime} \mathrm{P}_{2} \mathrm{~T}^{2} \mathrm{~T}^{1}$ ' sur un intervalle temporel $\mathrm{I}_{1}$, et, d'un autre côté, avec un repérage, noté par ' $\epsilon$ ', entre instants et entre rôles énonciatifs. Le schème général de la prise en charge énonciative dans le référentiel énonciatif REN, est une expression applicative, composée d'opérateurs appliqués à des opérandes 18 .

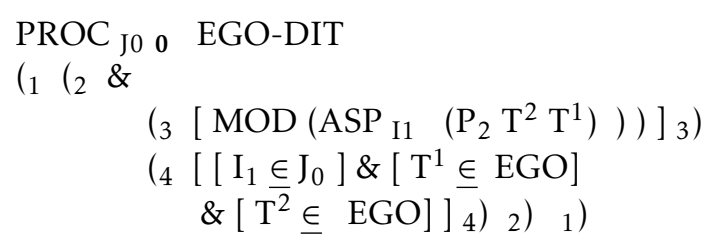

Son interprétation est: "à chacun des instants de $\mathrm{J}_{0}$, l'énonciateur EGO est en train de dire que ' $\mathrm{P}_{2} \mathrm{~T}^{2} \mathrm{~T}^{1}$ ' est énoncé et pris en charge selon diverses modalités qui portent sur son aspectualisa- tion actualisée sur $\mathrm{I}_{1}$, repéré par rapport à l'intervalle d'énonciation $\mathrm{J}_{0}$, les actants $\mathrm{T}^{1}$ et $\mathrm{T}^{2}$ étant repérés par rapport à EGO». Ce schème énonciatif fait apparaître deux opérandes: la relation prédicative aspectualisée ' $\left(3 \cdots_{3}\right)^{\prime}$ ' coordonnée à ses coordonnées énonciatives (relations temporelles et inter-sujets) ' 4 $\left.\ldots_{4}\right)^{\prime}$.

A titre d'exemple, la signification aspectuo-temporelle de l'énoncé déclaratif Il me conduit à l'école est décrite comme suit: la relation prédicative est un processus inaccompli, qui s'actualise sur l'intervalle $\mathrm{J}_{1}$, concomitant au processus énonciatif, d'où l'identification $\left[\mathrm{d}\left(\mathrm{J}_{1}\right)=\mathrm{d}\left(\mathrm{J}_{0}\right)\right]$ entre les bornes droites ouvertes, ou plus formellement:

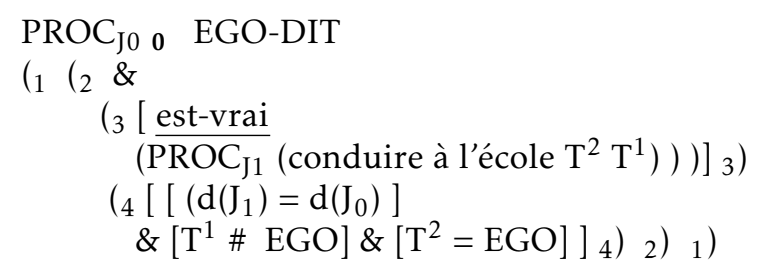

Dans cette représentation, le sujet il et le complément d'objet me sont les traces linguistiques des repérages inter-sujets respectifs $\left[\mathrm{T}^{1} \#\right.$ EGO] et $\left[\mathrm{T}^{2}=\mathrm{EGO}\right]$.

Dans la théorie de l'énonciation, les relations dialogiques prennent toute leur importance, en particulier lorsqu'elles traitent de la vérité, car:

$$
\text { («...) je ne m'élève de la certi- }
$$

\footnotetext{
17 J.-P. Desclés, « Opérations et opérateurs énonciatifs », in Colas-Blaise et al. (eds), 2016, o.c., pp. 69-88.

18 Ce schème est formulé ici dans un style analogue au langage de programmation LISP, en utilisant, pour faciliter la lecture, des parenthèses ouvrantes et fermantes numérotées.
} 
tude de la vérité qu'associé; ou la vérité n'advient, ou plutôt se reconnaît, jamais solitaire mais par consensus par accord avec qui je poursuis ce dialogue et non " tous les autres » qui ne suivent pas la discussion.

(F. Jullien, Si parler va sans dire, Paris, Seuil, 2006)

Pour prendre en compte ce genre de point de vue dialogique, il devient indispensable de complexifier la « logique moderne ", qui a su, avec G. Frege (1893), formaliser la prise en charge, par un énonciateur universel, de la vérité d'une proposition ' $p$ ', captée par l'opérateur frégéen de l'assertion universelle p' ( $\mathrm{p}$ est vraie »). On peut comparer cette assertion universelle de la logique moderne, avec l'assertion relative à un énonciateur EGO qui l'actualise sur un intervalle temporel d'énonciation (qualitatif) $\mathrm{J}_{0}$ :

\section{PROC $_{\text {Jo }}($ EGO-DIT (est-vrai (p))}

Ce schème d'assertion signifie que ce qui est déclaré vrai pour EGO dans un certain espace temporel, ne l'est pas nécessairement pour son coénonciateur ou pour un autre énonciateur potentiel distinct de EGO. Ce qui est essentiellement visé par G. Frege et, à sa suite, par la logique moderne, c'est l'étude de la vérité des énoncés atem- porels (des théorèmes), qui, de par leurs démonstrations mathématiques ou logiques, sont des énoncés indépendants de ceux qui les énoncent et par conséquent universels (du moins dans le cadre d'une théorie mathématique ou logique précise). On peut vouloir élargir l'horizon de la logique frégéenne en visant une étude plus complexe des raisonnements dialogiques, pas seulement en philosophie mais également dans les sciences, où s'affrontent, parfois à partir de points de vue opposés, énonciateur et coénonciateur insérés dans des contextes temporels et parfois spatiaux déterminés: "ce qui est vrai en deça des Pyrénées est faux au-delà » constatait Blaise Pascal.

Toute situation qui dénote une proposition aspectualisée est actualisée dans un référentiel sous la forme d'un état (inaccompli) ou d'un événement (accompli ou achevé, selon les cas) ou encore d'un processus (par nature inaccompli). Ces trois notions aspectuelles sont utilisées chez des linguistes comme B. Comrie et J. Lyons; ils sont, d'un point de vue cognitif, beaucoup plus naturels et intuitifs que les distinctions " state ", " activity ", " accomplishment » et «achievement» de Z . Vendler ${ }^{19}$, pourtant largement reprises par un grand nombre d'aspectologues, sans toutefois recevoir des définitions claires, étant présentées seulement à l'aide d'exemples; or, l'exemple seul ne

19 Z. Vendler, "Verbs and times", Philosophical Review, 66, 1957, pp. 143-160. 
suffit pas à « définir un concept».

Les concepts aspectuels d'état, d'événement et de processus sont appréhendés sous la forme de trois opérateurs aspectuels élémentaires, qui, en s'appliquant sur un opérande (un dictum ou une lexis, c'est-à-dire une relation prédicative), l'actualisent sur des intervalles continus d'instants dont les propriétés topologiques sont différen-

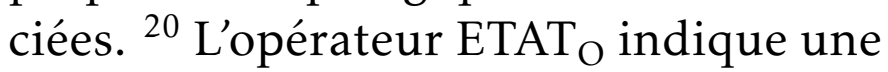
stabilité de l'opérande durant un intervalle topologique $\mathrm{O}$ " ouvert », c'està-dire sans que ni un premier instant ni un dernier instant appartiennent à l'intervalle ouvert $\mathrm{O}$ d'actualisation de l'état. L'opérateur $\mathrm{EVEN}_{\mathrm{F}}$ indique que son opérande est une transition événementielle entre deux états, cette transition est actualisée sur l'intervalle topologique «fermé » $F$, auquel appartiennent le premier instant et le dernier instant d'actualisation de l'événement. L'opérateur processuel $\mathrm{PROC}_{\mathrm{J}}$ actualise une modification en cours d'au moins l'un des actants de l'opérande, sur un intervalle semi-ouvert J, qui possède, à gauche, une borne fermée (l'instant initial de la modification) et, à droite, une borne ouverte, signifiant ainsi que le processus en cours n'a pas de dernier instant d'actualisation, étant inaccompli. Ces trois opérateurs aspectuels de base ne sont pas indépendants. En effet, un événement est toujours encadré par deux états, celui qui lui est antérieur et celui qui lui est postérieur; un état borné est encadré par l'événement qui fait entrer dans l'état et par l'événement qui en fait sortir; un processus qui s'accomplit atteint un dernier instant d'actualisation, il engendre alors, à ce dernier instant d'actualisation, un événement. Un événement engendré par l'accomplissement d'un processus peut être simplement accompli ou éventuellement achevé car complet, comme l'exemplifie la distinction sémantique entre les deux énoncés suivants: Luc a écrit toute la matinée [événement accompli] / Luc a écrit son compterendu en une heure [événement achevé, donc accompli]. Beaucoup d'approches formelles de l'aspectualité considèrent que l'événement est « ponctuel »; nous sommes en désaccord avec cette caractérisation ainsi que le montre en particulier l'exemple Il a gagné la partie d'échec en dix minutes. Un événement accompli d'une relation prédicative engendre en même temps un état résultatif, postérieur et contigu à l'événement, cet état affectant, après l'actualisation accomplie de l'événement, l'un des actants de la relation prédicative. Les différentes valeurs aspectuelles, souvent catégorisées par des jeux spécifiques de morphèmes grammaticaux, sont des spécifications de ces trois valeurs de base; ces spécifications s'organisent

\footnotetext{
${ }^{20}$ J. P. Desclés et Z. Guentchéva, "Universals and Typology”, in R. Binnick (ed), Oxford Handbook of Tense and Aspect, New York, Oxford University Press, 2012, pp. 123-154.

21 J.-P. Desclés, “A cognitive and conceptual approach to tenses and aspect markers", in Z. Guentchéva (ed.) Aspectuality and
} 
dans une carte sémantique. ${ }^{21}$ Exemplifions quelques-uns de ces opérateurs aspectuels:

(a) Luc signe en ce moment un document de reconnaissance de dette.

[Processus actuel, inaccompli];

(b) Hier, Luc signait le document lorsque son avocat est entré brusquement.

[Processus non actuel, inaccompli à l'instant du début de l'événement suivant, considéré comme sécant];

(c) Hier, Luc a lu le document, il l'a signé puis il a quitté aussitôt la salle.

[Succession de trois événements accomplis];

(d) Luc a maintenant signé le document, tout le monde en est soulagé.

[État résultant actuel inaccompli d'un événement accompli].

A ces gloses métalinguistiques correspondent différents diagrammes temporels que, faute de place, nous ne pouvons pas présenter ici.

\section{Primitives en sémantique cognitive}

Les significations des unités prédicatives (verbes) et des opérateurs prépositionnels et préverbaux sont représentables par des schèmes interprétatifs, qui résultent de compositions de quelques " primitives » sémantico-cognitives. En effet, comme le rappelle Blaise Pascal, toute description suppose des primi- tives:

"Cette véritable méthode [la méthode géométrique] (...) consisterait en deux choses principales: l'une, de n'employer aucun terme dont on n'êेt auparavant expliqué nettement le sens; l'autre de n'avancer jamais aucune proposition qu'on ne démontrât par des vérités déjà connues; c'est-à-dire, en un mot, à définir tous les termes et à prouver toutes les propositions. (...)»

" (...) Aussi, en poussant les recherches de plus en plus on arrive nécessairement à des mots primitifs qu'on ne peut plus définir, et à des principes si clairs qu'on n'en trouve plus qui le soient davantage pour servir à leur preuve. »

"(...) Car il n'y a rien de plus faible que le discours de ceux qui veulent définir ces mots primitifs. »

(Blaise Pascal: De l'esprit géométrique et de l'art de persuader, in Euvres complètes, Paris, Gallimard, 1914, p. 577578).

Les primitives sémantiques ne sont pas nécessairement réduites à de simples sèmes classificatoires, comme le 
laissait entendre la linguistique structurale lorsqu'elle procédait à des analyses de familles d'entités nominales (comme chaise / fauteuil / tabouret canapé...), en s'inspirant des décompositions platoniciennes de Porphyre.

Une description sémantique plus élaborée à l'aide de plus d'une cinquantaine de primitifs susceptibles d'être communs à toutes les langues est entreprise par A. Wierzbicka22, Une telle analyse pose cependant un certain nombre de problèmes qui ne seront pas discutés ici. On peut également citer le dictionnaire syntaxique et sémantique d'un nombre très important de verbes polyémiques français de $\mathrm{K}$. Bocgacki et al. ${ }^{23}$, qui utilise 15 primitives de base.

Dans notre approche de la sémantique cognitive, nous proposons un système réduit de primitives structurantes qui sont non seulement des sortes (ou types de base) d'entités ayant un ensemble de caractéristiques communes (exemple: types des objets individuels, des objets méréologiques, des classes distributives, des classes collectives, des lieux... ) mais aussi différents types fonctionnels d'opérateurs (et de relateurs), récursivement engendrés à partir des types de base; en se composant, les instances (des opérateurs et des relateurs) de ces types fonctionnels constituent les primitives structurantes chargées de construire, en se composant entre elles, différents schèmes interprétatifs, plus ou moins complexes, destinés à représenter des significations d'unités linguistiques (grammaticales et verbales).24

Les types fonctionnels sont des types de Church (1940) définis à partir d'un ensemble de types de base (ou sortes) par les règles:

- les types de base sont des types fonctionnels;

- si $\alpha$ et $\beta$ sont des types fonctionnels, alors $\mathbf{F} \alpha \beta$ est un type fonctionnel, c'est-à-dire un type d'opérateurs qui s'appliquent seulement à des opérandes de type $\alpha$ pour construire des résultats de type $\beta$.

Les catégories syntaxiques représentées dans les grammaires catégorielles (d'Ajdukiewicz, de Bar-Hillel, de Lambek...) par des types sont des types de Church 25, contenant une information supplémentaire sur l'orientation, à droite ou à gauche, de la position attendue de l'opérande, de façon à tenir compte des contraintes de l'ordre linéaire syntagmatique.

Un grand nombre des primitives structurantes sont en correspondance assez directe avec des représentations

\footnotetext{
22 A. Wierzbicka, «La quête des primitifs sémantiques ». Langue française, n 98, Les primitifs sémantiques, 1993, pp. 9-23.

$23 \mathrm{~K}$. Bogacki et al., Dictionnaire sémantique et syntaxique des verbes français, Warszawa, Panstwowe Wydawnictwo Naukowe, 1983.

${ }^{24}$ Voir J-P. Desclés, G. Guibert et B. Sauzay, Calculs des significations par une logique d'opérateurs * Vers une logique d'opérateurs; ${ }^{* *}$ Concepts et schèmes analysés par la logique combinatoire, Vol. II, Toulouse, Cépaduès, 2016, pp. 418-493.

25 Voir J.-P. Desclés, "Brève généalogie des grammaires catégorielles”, Verbum, tome XL, N 2, 2018, pp. 143-172; J.P. Desclés et al., Logique combinatoire et $\lambda$-calcul, Vol. I, Toulouse, Cépaduès, 2016, pp. 201-234; voir aussi Vol. II, 2016, o.c., pp. $304-355$.
} 
cognitives abstraites et organisées par les activités cognitives de perception et d'action relatives au monde externe au langage. C'est le cas, par exemple, des types de base: les distinctions entre objets individuels ou méréologiques, entre lasses distributives ou collectives, entre un objet et la position qu'il occupe dans un lieu, sont organisées principalement par la perception.

Les primitives structurantes relevant des types de base et des types fonctionnels sont des éléments nécessaires (mais souvent pas suffisants) pour décrire les significations des unités grammaticales, nominales et verbales des langues. D'autres primitives technico-culturelles viennent s'ajouter, dans les descriptions des significations, aux primitives structurantes de l'activité de langage. Par exemple, la notion sémantique de " roue » est seulement présente dans les cultures qui ont acquis cette technologie et, dans ce cas, la description de la signification d'unités lexicales polysémiques (le carrosse roule / rouler dans un fossé / se rouler dans une couverture / rouler un tapis / rouler quelqu'un / ça roule ...) pourra exploiter cette primitive technico-culturelle dans leurs lexicalisations.

La sémantique cognitive repose sur une hypothèse très générale, souvent laissée implicite dans la plupart des approches, formulée grossièrement et rapidement comme suit: il existe une certaine interaction entre l'activité de langage et les activités cognitives de perception de l'environnement et d'action (plus ou moins intentionnelle) sur cet environnement. Une telle hypothèse revient à affirmer une forme d'ancrage des primitives structurantes, constitutives des significations, sur les catégorisations cognitives structurées par la perception et l'action; ces primitives structurantes font partie des invariants sémantiques de l'activité de langage. Cet ancrage n'est cependant pas une "réduction " du langage à la seule perception; en effet, l'activité de langage des humains leur donne la capacité de s'abstraire du vécu accessible à la seule perception, pour dégager des primitives cognitives générales qui transcendent différents domaines (la spatialité, la temporalité, des domaines d'action et d'activité). Ce type d'abstraction cognitive entraîne une véritable économie des systèmes sémiotiques des langues, notamment en structurant une polysémie d'un grand nombre de termes linguistiques, ce qui évite ainsi aux locuteurs de devoir recourir à une multitude de plus en plus importante de termes linguistiques différents, créés pour chaque nouvelle situation singulière, ce qui reviendrait alors à empêcher la formation de langues, outils fondamentaux de socialisation des communautés humaines. Pour G. Frege, la langue n'est qu'un "habit de la pensée »; à sa suite les logiciens modernes voient dans la polysémie des termes linguistiques un défaut inhérent aux langues, 
défaut qu'il convient, pour raisonner correctement, de savoir " rectifier » en construisant et en utilisant un " langage de la pensée » destiné à éviter ainsi le flou et l'imprécision véhiculées par les langues. Contrairement à cette propriété négative attribuée aux langues, nous défendons l'idée inverse: avec la possibilité de créer un réseau polysémique structuré de significations associées à une même forme signifiante, avec souvent un invariant sémantique abstrait (un "signifié de puissance " au sens de G. Guillaume), les langues sont munies d'une propriété positive qui leur donne la capacité sémiotique d'une adaptation économique et continuelle à la verbalisation de nouvelles situations. Pour la théorisation linguistique et les descriptions qui s'en déduisent, les linguistes doivent expliquer et décrire comment les langues gèrent la polysémie inhérente aux langues.

Remarque: Dans plusieurs articles, nous avons présenté des exemples de traitement de polysémies lexicales et grammaticales, par exemple celle du verbe donner et celle des morphèmes grammaticaux d'aspect et de temps, en faisant appel à des primitives structurantes.

Outre les différentes sortes d'entités (objets individuels, classes distributives et collectives, lieux etc.), les trois valeurs du repérage abstrait $\{=$, $\neq$, \#\} sont des exemples importants de primitives structurantes statiques, ancrées sur la perception directe de l'environnement spatial, puis généralisées, par abstraction, à d'autres do- maines moins directement accessibles à la perception. Ces trois opérateurs génériques d'identification (=), de différenciation $(\neq)$ et de rupture (\#) se composent avec les opérateurs topologiques qui spécifient l'intériorité, l'extériorité, la frontière et la fermeture d'un lieu. Ces opérateurs topologiques avec le repérage sont des primitives structurantes qui interviennent dans la construction de nombreux schèmes interprétatifs.

Présentons d'autres primitives structurantes, à savoir des primitives cinématiques et dynamiques. Le mouvement, noté MOUVT, d'un objet qui passe d'une localisation à une autre et le changement, noté CHANGT, des états d'un même objet, sont des primitives cinématiques qui relient deux situations; la Physique d'Aristote avait déjà souligné leur importance. Dans le domaine de l'action, l'effectuation d'une action, notée FAIRE (à ne pas confondre avec le verbe faire du français), établit une relation dynamique entre une entité et un changement ou un mouvement, qui affecte un objet. L'entité effectuant une action peut être un agent ou un instrument, placé alors sous le contrôle d'un agent, ou encore une entité physique externe (comme la pluie, l'orage...). La notion d'agent sousjacente à la signification d'un énoncé comme Adam mange la pomme que lui tend Ève, est représentée par la primitive de contrôle, notée CONTR, caractérisée par « une mise en œuvre du 
déclenchement ou de l'interruption de l'effectuation d'une action »; c'est une capacité exercée par les agents mais pas par les instruments (ex. Le train transporte des voyageurs), pas plus que les forces naturelles (ex. Les éclairs transpercent les nuages). Les primitives d'effectuation et de contrôle d'une effectuation sont les constituants de schèmes dynamiques avec ou sans agents; elles ne doivent pas être confondues avec la primitive de causalité, notée CAUSE, reliant deux situations cinématiques ou dynamiques complètes (exemple: Le réchauffement du climat entraîne la montée des océans). La primitive de visée téléonomique, notée TELEO, met en relation un agent intentionnel avec la représentation d'une situation visée et non encore actualisée, le but de son action (ex. Luc envoie sa déposition au juge d'instruction).26 Ces primitives (statiques, cinématiques, dynamiques, causales, téléonomiques) se composent entre elles pour construire des relations portant sur des relations déjà établies, sous le forme de schèmes sémanticocognitifs qui représentent les significations d'unités prédicatives, mais aussi des prépositions et des préverbes qui se composent avec des unités verbales...

\section{Topologie et Quasi-topologie}

Tel que nous l'entendons, un schème sémantico-cognitif a une signification analogue au schème de Kant; c'est un intermédiaire entre le sensible (accessible à la perception) et le concept qui trouve une expression sémiotique sous la forme d'une unité linguistique:

"Kant exige, pour rendre possible l'application des concepts purs de l'entendement aux intuitions sensibles, un tiers, un moyen terme grâce auquel les deux autres, bien qu'absolument hétérogènes peuvent coïncider - et il trouve cette médiation dans le schème transcendantal qui, d'une part, est intellectuel, et, de l'autre sensible. »

(E. Cassirer, La philosophie des formes symboliques, Paris, Editions de Minuit, 1953, p.154).

Les schèmes sémantico-cognitifs (SSC) exprimés par des formes symboliques sont souvent associés à des schèmes plus figuratifs et iconiques, et même, dans certains cas, à des images directement liées à une perception d'un environnement ou à une action opérant dans cet environnement. Le processus de compréhension d'une expression

\footnotetext{
${ }^{26}$ Les primitives relationnelles de mouvement, de changement, et de contrôle, ainsi que les notions de schèmes statiques, cinématiques et dynamiques, ont été introduites dans J.-P. Desclés, «Représentation des connaissances: archétypes cognitifs, schèmes conceptuels, schémas grammaticaux », Actes sémiotiques, Documents VII, N 69-70, EHESS-CNRS, 1985, pp. 5-51; voir aussi Desclés et al., volume II, 2016, o.c., pp. 451-496.
} 
linguistique opère une construction d'une représentation symbolique, avec d'éventuels transferts vers des représentations plus figuratives et iconiques, en aval de la décomposition analytique des opérations énonciatives; inversement, un processus de production langagière opère, à la suite d'un transfert éventuel de représentations figuratives, sur des représentations symboliques formulées par des schèmes instanciés et spécifiques à une langue, ces représentations symboliques sont ensuite transformées, en plusieurs étapes, par des mécanismes de synthèse intégrative, jusqu'à obtenir un agencement syntagmatique d'unités linguistiques agencées dans des énoncés proposés à un échange verbal.

L'ancrage (et non pas la réduction) de la sémantique du langage sur les activités cognitives de perception et d'action fait naturellement surgir une question théorique: la représentation spatiale est-elle première dans l'activité de langage, les représentations non spatiales en étant dérivées, par exemple par des processus métaphoriques? E. Kant a pris position sur cette question:

"L'espace est une représentation nécessaire a priori qui sert de fondement à toutes les intuitions extérieures. (...) Il est donc considéré comme la condition de possibilité des phénomènes, et non pas comme une détermination qui en dépende, et il est une représentation a priori qui sert de fondement, d'une manière nécessaire, aux phénomènes extérieurs »

(E. Kant, Critique de la raison pure, Paris, Presses Universitaires de France, 1781/1944, p. 56)

En d'autres termes, les représentations opérées par les langues seraient d'abord ancrées sur les représentations spatiales, renouant ainsi avec l'ancienne hypothèse " localiste " des Stoïciens et réactualisée à notre époque, par, entre autres, R. Jackendoff et tous ceux (philosophes et linguistes) qui défendent la primauté de l'espace, en se réclamant de Kant. Cette position est cependant loin d'être partagée par la communauté des linguistes. Par exemple, G. Guillaume et B. Pottier, jugent que la temporalité serait beaucoup plus fondamentale que la spatialité. Pour notre part, nous reformulons la question précédente: pour construire des représentations ayant une vocation explicative, peut-on avoir recours à des représentations qui seraient non spatio-temporelles? G.-G. Granger ${ }^{27}$ évoque de telles formes de représentations explicatives qui n'ont pas un support nécessairement spatial. De nombreux logiciens (par exemple, R. Blanché) raisonnent sou-

27 G.-G. Granger, formes, opérations, objets, Paris, Vrin, 1994. 
vent avec des « carrés » et des " hexagones » sémiotiques de propositions liées a cette structure. Le mathématicien $E$. Weil a su faire émerger des structures algébriques de groupes à partir des organisations de parenté analysées en anthropologie par C. LéviStrauss ${ }^{28}$. La théorie des graphes décrit et démontre les propriétés mathématiques de nombreuses situations géométriques (comme les labyrinthes) et non géométriques d'algorithmes qui opèrent avec des représentations formalisées par des points et des flèches. A un niveau beaucoup plus abstrait, la théorie mathématique des catégories de S. Eilenberg et S. Mac Lane (1945) travaille avec des morphismes entre objets structurés, formant une catégorie, et avec des foncteurs entre de telles catégories, pour expliquer les concepts les plus profonds des mathématiques ${ }^{29}$.

Examinons maintenant une seconde question. En tant que théoricien d'une approche plus européenne du courant actuel de la sémantique cognitive, $\mathrm{B}$. Pottier a abstrait d'un grand nombre d'analyses sémantiques le schème général du trimorphe, qui est, selon nous, un exemple d'un schème cognitif général:

"Le trimorphe est une représen-
tation continue et triphasée d'un
événement ou d'une catégorisa-
tion»

«(..) Un schème n'est pas une représentation spatiale même si généralement il occupe une étendue, car il se déroule également dans le temps (il est mouvement), et son abstraction de toute aire d'instanciation le rend apte à se réaliser librement. »

(B. Pottier, Représentations mentales et catégorisations linguistiques, 2000, p. 143)

Il apparaît ainsi nettement, par le biais de ce schème du trimorphe, que les différentes phases d'un événement sont liées à des modifications continues, ou discontinues d'états, constitutives d'une transition globale entre deux états, l'un initial et antérieur, l'autre terminal et postérieur, exemplifiée par les différentes locutions verbales: se mettre puis commencer à écrire / être en train d'écrire et être en écriture / finir puis terminer d'écrire. Pour effectuer le parcours d'un événement transitionnel, il faut d'abord avoir quitté une stabilité antérieure, traverser une phase initiale de modification pour atteindre une phase d'activité, localisée à l'intérieur de la transition globale, ensuite traverser une phase terminale de modification afin d'atteindre une stabilité terminale postérieure à la transition. De nombreux verbes préfixés illustrent parfaitement certaines phases

\footnotetext{
28 Voir, par exemple, A. Warusfel, Les mathématiques modernes, Paris, Le rayon de la science, 1969, pp. 166-170.

${ }^{29}$ Voir F. W. Lawvere et S. H. Schanuel, Conceptual Mathematics. A first introduction to categories, Cambridge Univerity Press, 2009.
} 
d'une transition événementielle (ex. en français: aborder = aller vers le bord / exhiber $=$ faire sortir, faire apparaître / atteindre (une frontière de l'extérieur ou de l'intérieur : atteindre le toit / atteindre le plancher / atteindre le plafond). On peut évoquer les compositions en grec ancien de différents préverbes (souvent associés à des prépositions) avec les verbes, par exemple avec le verbe $\beta a v \omega \omega$ ('je marche') pour signifier, selon le préverbe choisi, 'monter', 'débarquer', 'traverser', 'sortir', 'monter dans', 'monter sur', 'descendre', 'passer de ... à', 'transgresser', 'avancer', 'arriver'. Les différentes phases d'un trimorphe représentent les " modes d'action » d'un procès (Aktionsarten); elles sont exprimées souvent en français au moyen de verbes auxiliaires alors que dans d'autres langues, par exemple les langues slaves, ce sont des préverbations, liées sémantiquement à des prépositions, qui marquent ces valeurs aspectuelles.

Le trimorphe de B. Pottier renvoie implicitement à une topologie abstraite (par nécessairement spatiale). Cette remarque soulève immédiatement la question: faut-il se restreindre aux concepts de la topologie générale étudiée par les mathématiques? Rappelons que ce qui est appelé " topologie générale » s'est élaborée pendant plus de deux siè- cles avec les travaux de philosophes et de mathématiciens (Leibniz, Euler, Cauchy, Weierstrass, Fléchet...) pour étudier, puis définir avec précision et de façon opératoire, des problèmes comme la continuité des fonctions ou la convergence, ou non convergence, de certaines suites récurrentes de nombres. Peuton entreprendre de complexifier l'étude des notions topologiques "classiques" pour les étendre aux représentations réclamées par l'étude sémantique des langues ? Peut-on manipuler des représentations figuratives (formulées dans certaines approches de sémantique cognitive) au moyen d'opérateurs logico-algébriques qui se prêteraient, à des calculs? Inversement, des représentations symboliques, sans aucune interprétation a priori géométrique, arriventelles à capter adéquatement et intuitivement les catégorisations " naturelles » qui exigent de prendre en compte d'une part, l'opposition entre l'extension et l'intension d'un concept et d'autre part, les opérations de détermination des objets, les exemplaires typiques et atypiques du concept, en évitant de confondre les atypiques avec les exceptions du concept ${ }^{30}$ ?

Leibniz, créateur de l'Analysis situs, reconnaissait les limites interprétatives des représentations algébriques:

\footnotetext{
${ }^{30}$ Voir J.-P. Desclés et A. Pascu, 'Logic of Determination of Objects: How to articulate 'Extension' with 'Intension' and 'Objects' with 'concepts' ", Logica Universalis 5/1, 2011, pp. 75-89; J-P. Desclés, A. C. Pascu,. "Logic of Typical and Atypical Instances of a Concept. | A Mathematical Model". Axioms, 8(3), 2019, p. 104 et sq. Ces deux approches proposent une analyse logique des problèmes de catégorisation, très différente de la la théorie usuelle des prototypes et de logique floue de L. Zadeh.
} 
"Car les caractères algébriques n'expriment pas tout ce qui doit être considéré dans l'espace : ils ne représentent pas directement la position (situs) des points, mais l'étudient par l'intermédiaire des grandeurs au prix de maint détour $»$.

(Leibniz Mathematische Schriften, p. 142)

G.-G. Granger, lui aussi, remarque:

"Il convient donc de rechercher une Charactéristique plus naturelle des phénomènes géométriques, dont la catégorie fondamentale ne sera pas la mesure des grandeurs, mais la position relative des objets immédiats que sont les «points » et les "figures. »

(G.G. Granger, formes opérations objets, Paris, Vrin, 1994, p. 213)

La topologie générale des mathématiciens présente l'avantage d'être essentiellement qualitative, puisque aucune métrique d'un espace euclidien n'est a priori nécessaire à la définition de concepts topologiques comme "voisinage d'un point ", " intérieur, frontière, fermeture ou extérieur d'un lieu ", " lieu ouvert ou fermé »...: "Penser le topologique plutôt que l'euclidien" est un conseil de Merleau-Ponty. L'alternance entre nombre et espace est soulignée par G.-G. Granger:

" L'idée de nombre et l'idée d'espace apparaîtront désormais dans l'histoire des mathématiques en un contrepoint qui tantôt donnera plus de force à l'orientation vers le nombre, tantôt à l'orientation vers l'espace. $\mathrm{Ce}$ que nous appelons orientation vers le nombre a du reste donné naissance à un traitement algébrique des objets qui met l'accent sur l'activité opératoire abstraite de la pensée. De sorte que l'on a pu mettre en vedette à différents moments une alternance d'un esprit algébrique et d'un esprit géométrique dans le développement mathématique de l'idée d'espace. ».

(G-.G. Granger, La pensée de l'espace, Paris, Editions Odile Jacob, 1999, p. 225)

Pour G. Th. Guilbaud, connu pour ses travaux orientés vers une approche mathématique des sciences humaines et sociales (économie, sociologie, psychologie, anthropologie et linguistique), la topologie est un langage qui décrit précisément «l'à peu près »:

"Les images géométriques sont d'un grand secours, parce que la structure topologique $y$ est, pour ainsi dire, donnée dans 
l'intuition. Mais il faut se méfier. Chaque fois que l'on veut introduire les procédures d'approximation sur une catégorie d'objets, il faut bien préciser de quelle espèce de topologie on va faire appel. Dans un grand nombre de problèmes d'origine géométrique, la topologie semble s'imposer; mais viendra le moment où l'on s'apercevra qu'il faut choisir une topologie et que le mathématicien dispose alors d'une certaine liberté »

« (...) L'Analyse Mathématique n'a pas hésité à récupérer "le langage commun. Elle aussi dit "PRESQUE ». Mais, comme disait Goethe, c'est tout autre chose! »

(G. Th. Guilbaud, Leçons d'à peu près, Paris, Christian Bourgeois, 1985, pp. 214-220)

Mais que disait Goethe?

"Les mathématiciens, c'est comme les Français, on leur parle, ils traduisent en leur langue, et ça devient, tout de suite, tout à fait autre chose ». (Goethe).

L'axiomatisation algébrique de $\mathrm{K}$. Kuratowski de la topologie associe à un lieu 'Loc' sa frontière 'Fro(Loc)' qui sépare son intérieur 'Int(Loc)' de son extérieur 'Ext(Loc)', la fermeture 'Fer(Loc)' étant l'union de son intérieur et de sa frontière:

$$
\begin{aligned}
& \text { Fer }(\text { Loc })==_{\text {def }} \\
& \underline{\text { Fro }}(\text { Loc })==_{\text {def }} \\
& \underline{\text { Fer }}(\operatorname{Loc}) \cup \underline{\text { Fro }}-\underline{\text { Int }}(\text { Loc })
\end{aligned}
$$

Les propriétés de l'opérateur 'Int' qui détermine l'intérieur d'un lieu 'Loc' sont, entre autres:

$$
\begin{aligned}
& \underline{\operatorname{Int}}(\mathrm{Loc}) \subseteq \text { Loc } \subseteq \text { Fer }(\text { Loc }) \\
& \overline{\operatorname{Int}}(\operatorname{Int}(\operatorname{Loc}))=\underline{\operatorname{Int}}(\overline{\operatorname{Loc}}) \\
& {\left[\operatorname{Loc}_{1} \subseteq \operatorname{Loc}_{2}\right]=>\left[\underline{\operatorname{Int}}\left(\operatorname{Loc}_{1}\right) \subseteq \underline{\operatorname{Int}}\left(\operatorname{Loc}_{2}\right)\right]}
\end{aligned}
$$

L'alternance géométrique/algébrique est parfaitement illustrée par les 7 propositions modalisées positives du système $\mathrm{S}_{4}$ de Lewis des modalités 'il est nécessaire' et 'il est possible'; L'organisation de ces 7 propositions modalisées est en étroite correspondance avec les 7 lieux topologiques distincts pouvant être associés à un lieu donné selon l'algèbre de Kuratowski des opérateurs topologiques 'prendre l'intérieur' et 'prendre la fermeture', auxquels sont associés les opérateurs modaux respectifs du 'nécessaire' et du 'possible'. 31

R. Thom 32 a montré comment la théorie topologique des singularités élémentaires (des catastrophes), qui sont des ruptures brusques dans les changements continus, pouvait prendre le re-

\footnotetext{
${ }^{31}$ Voir M. Barbut, «Topologie générale et algèbre de Kuratowski », Mathématiques et sciences humaines, 1965, pp. 11-27; J.-B. Grize, Logique moderne III, Paris, Gauthier-Villars, 1973, pp. 37-38.

32 R. Thom, Stabilité structurelle et morphogénèse, John Benjamins, 1973.
} 
lais sémantique de la théorie syntaxique des schémas prédicatifs actanciels (stemmas) de L. Tesnière $e^{33}$; il a présenté quelques schèmes figuratifs qui représentent les significations prototypiques de quelques prédicats verbaux, comme donner à en français. Cette application de la " théorie des catastrophes » à la morphogénèse et à la sémantique a inspiré plusieurs recherches menées, en sémiotique par J. Petitot, et en sémantique par B. Victorri et W. Wildgen ${ }^{34}$. A. Culioli invoque également la topologie pour certaines analyses linguistiques. ${ }^{35}$

La signification des petits mots grammaticaux encore / pas encore / déjà / déjà plus, utilisés aussi bien dans l'analyse de la traversée d'un lieu spatial (Il n'est pas encore à Rio / Il est déjà dans Rio / Il est vraiment dans Rio / Il est encore dans Rio / il n'est déjà plus à Rio) que dans les divers ajustements d'une formulation juridique (Cette proposition de loi n'est pas encore légale / elle est déjà légale / elle est complètement légale / elle est encore légale / elle n'est déjà plus légale / elle est illégale), nous a amené à " dépasser » le cadre trop étroit de la topologie générale. L'analyse des marqueurs de temporalité et de l'aspectualité nous a conduit à définir les propriétés mathématiques d'une quasi-topologie pour analyser et donner des représentations formelles de certains problèmes que les sciences humaines abordent. Pour représenter les " catégorisations naturelles » 36, souvent verbalisées par les langues, ma collègue Anca Pascu et moi-même, nous mettons en place une «quasi-topologie » dans laquelle un lieu muni d'une structure quasi-topologique 'Loc' est appréhendé avec sa frontière interne 'Fro-int(Loc)', et sa frontière externe 'Fro-ext (Loc)', d'où son intérieur strict 'Int-strict (Loc)' contenu dans son simple intérieur 'Int(Loc)' et sa fermeture large 'Fer-large (Loc)' qui contient sa simple fermeture 'Fer(Loc)'; les opérateurs quasi-topologiques possèdent les propriétés suivantes:

$$
\begin{aligned}
& \underline{\text { Int-strict }}(\text { Loc }) \subset \underline{\text { Int }}(\text { Loc }) \subseteq \text { Loc } \\
& \subseteq \underline{\text { Fer }}(\overline{\text { Loc }}) \subset \underline{\text { Fer }^{-} \text {-large }}(\mathrm{Loc}) \\
& \text { Fro-int }(\text { Loc })=\bar{d}_{\text {def }} \text { Fer }(\text { Loc })-\text { Int-stict }(\text { Loc }) \\
& \overline{\text { Fro-ext }}(\text { Loc })=_{\text {def }} \overline{\text { Fer-large }}(\text { Loc })-\underline{\text { Int }}(\text { Loc })
\end{aligned}
$$

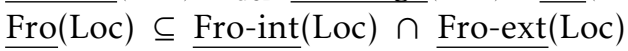

La traversée d'un lieu quasitopologique à partir de son extérieur implique plusieurs phases : entrer dans le lieu en traversant sa frontière externe puis sa frontière interne pour atteindre son intérieur strict, ensuite, traverser sa

\footnotetext{
${ }^{33}$ L. Tesnière, Éléments de syntaxe structurale, Paris, Klincksieck, 1959/ 1988.

${ }^{34}$ Par exemple, W. Wildgen, Catastrophe theoretic semantics. An application and elaboration of René Thom'theory, Amsterdam, John Benjamins. 1982.

35 Voir, par exemple A. Culioli, Variations sur la linguistique, 2002, o. c., p. 126 et p. 218.

36 A. C. Pascu, J-P. Desclés, I. Biskri, "A topological approach for the notion ofQuasi-topology structure" . South American Journal of Logic, Vol. X, n. X, 2019, pp. 1-18.

37 Voir J.-P. Desclés et Z. Guentchéva, «Quasi topological representations (QTR) of spatial places and spatio-temporal movements in natural languages ", in G. Marotta et al., Space in Language, Proceedings of the Pisa International Conference, Edizioni ETS, Firenze, 2010, pp. 213-233.
} 
frontière interne puis sa frontière externe pour finalement sortir du lieu. 37

La prise en compte des frontières interne et externe d'un même lieu, qu'il soit spatial, temporel, d'activité, cognitif ou notionnel, conduit à une plus grande finesse des analyses sémantiques, d'où résultent différents genres de schèmes des changements et des mouvements: (1) le schème de la tension binaire de G. Guillaume, avec ses deux régions qui convergent en un seul point de renversement; (2) le schème du trimorphe de B. Pottier, déjà mentionné, avec ses trois régions: un "avant ", un " pendant » et un "après "; (3) le schème topologique avec ses deux frontières et ses cinq régions intermédiaires; (4) le schème quasi-topologique avec ses frontières interne et externe (initiales et terminales) et la traversée de sept régions.

G. GUILLAUME :

Tenseur binaire :

Particularisation /

Universalisation

B.POTTIER :

Schème du trimorphe

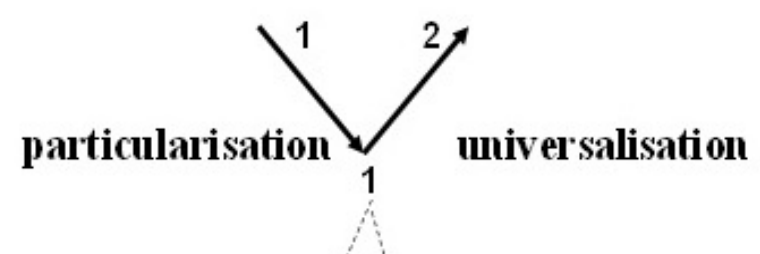

Schème des lieux abstraits top ologiques
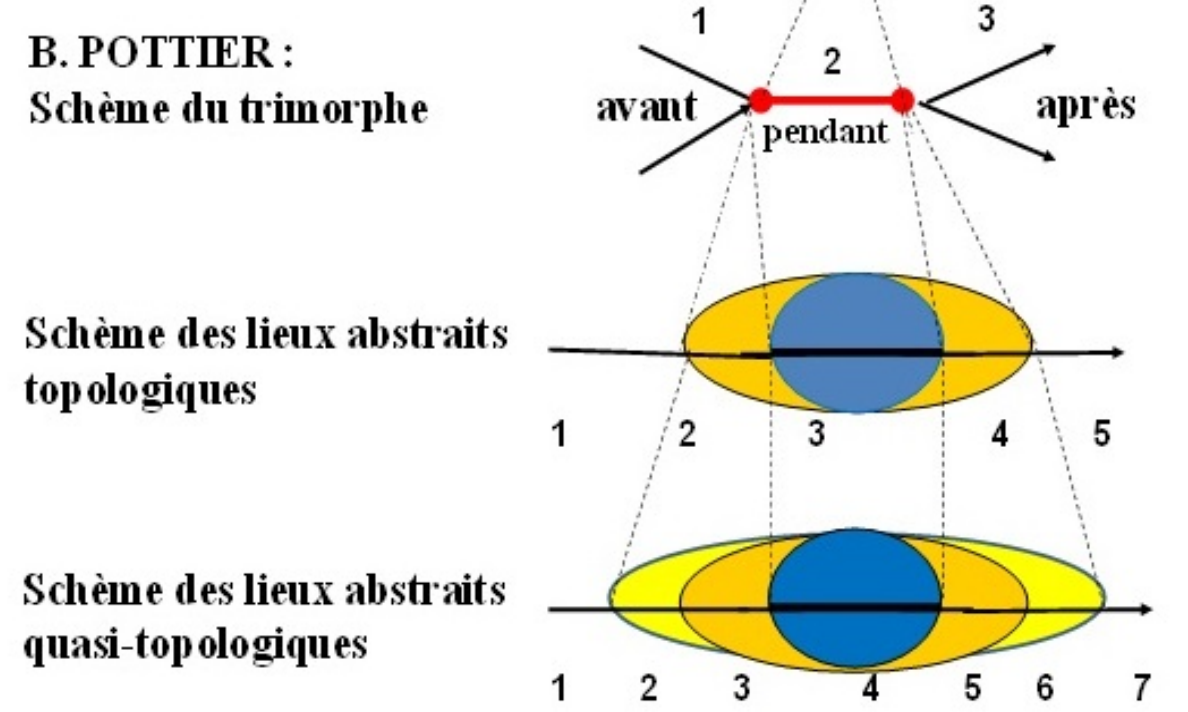

Figure: Différents schèmes avec leurs différentes phases dans la traversée d'un lieu

Ce genre d'application de la topologie et de la quasi-topologie à l'analyse des significations en sémantique des langues et en anthropologie $e^{38}$, avec leurs propriétés mathématiques, fait émerger un nouveau problème: com-

\footnotetext{
${ }^{38}$ La notion cognitive de « la nuit ", pensée et vécue par plusieurs cultures, s'analyse avec des concepts quasi-topologiques; voir J. P. Desclés, "La nuit pensée et vécue comme une frontière quasi-topologique », Ateliers d'anthropologie, Laboratoire d'ethnologie et de sociologie, Université de Nanterre, 2019 (à paraître en 2020).
} 
ment expliquer et décrire formellement le passage d'une représentation figurative (comme une représentation figurative par une " catastrophe ") à son expression verbale dans une langue? Par exemple, comment expliquer le passage du schème figuratif de "donner à » de R. Thom à l'unité prédicative, 'donner-à $(\mathrm{x}, \mathrm{y}, \mathrm{z})$ ' puis au schéma syntaxique ' $\mathrm{x}$ donne $\mathrm{y} a ̀$ ' $z$ ', avec ses trois arguments ayant des rôles actanciels différents, et comment tenir de la polysémie de ce verbe auquel sont associés plusieurs autres schèmes interprétatifs ? ${ }^{39}$ La même question se pose pour les représentations iconiques proposées par R. Langacker, B. Pottier ou par L. Talmy.

\section{Comment composer des opérateurs?}

Les représentations des significations sous la forme de représentations iconiques, figurative ou sous forme de diagrammes, restent insuffisantes pour entreprendre de comparer les méthodes de la linguistique contemporaine aux méthodes des sciences, dites dures. En physique classique, depuis Galilée et Newton, les mouvements dans l'espace d'un objet, comme la chute des corps, sont représentés par des représentations figuratives auxquelles ont été associées systématiquement des représen- tations algébriques avec une formulation d'équations qui, pour être résolues, ont fait appel à des calculs formels nouveaux (par exemple, des calculs sur les quantités infinitésimales). Les résultats de ces calculs ont été ensuite confrontés aux observations effectuées par divers procédés expérimentaux parfois originaux (comme le plan incliné de Galilée), afin de pouvoir valider les conceptualisations théoriques sous-jacentes aux calculs des d'équations. Pour sortir d'une étape "pré-galiléenne », trop enfermée dans une métaphysique néoaristotélicienne, et devenir une véritable science selon les canons des sciences de la nature, la linguistique, lorsqu'elle vise à expliquer et à faire comprendre ce qu'est l'activité de langage, se doit d'associer aux représentations figuratives des significations (par exemple, les représentations de donner de R. Thom, de R. Langacker ou de B. Pottier), des formulations symboliques intégrées dans des systèmes où des calculs logico-algébriques peuvent alors être effectués; c'est à ce prix que l'on pourra relier des représentations mentales (ou cognitives) (par nature, non directement observables) aux formes observables des énoncés, des textes et des discours. Pour cela, il faut disposer d'un formalisme bien fondé où tels calculs symboliques peuvent voir le jour. Pour de nombreux logiciens et

\footnotetext{
${ }^{39}$ Sur la polysémie verbale, voir J. P. Desclés, « Le problème de la polysémie verbale : donner en français », Cahiers de lexicologie, 2011-1, n 98, pp. 95-111.
} 
philosophes, la logique moderne, c'està-dire le calcul classique des propositions, des prédicats et des quantificateurs, héritage de Frege, Peano, Russell, a été «le » formalisme privilégié, devenant l'outil intellectuel du développement d'une philosophie analytique du langage. Les réponses proposées par la logique moderne a cependant montré ses limites avec une analyse approfondie et adéquate des langues naturelles. Remarquons également que depuis G. Frege, la logique, qu'il a mathématisée de façon très intéressante en formalisant les prédicats et les quantificateurs comme des fonctions mathématiques non numériques, a éliminé toutes les opérations de détermination, dont les traces sont les adjectifs, les relatives (déterminatives et explicatives) et la présence de divers articles (dans les langues qui en font usage), au profit des seules opérations de prédication et de quantification. Pourtant, antérieurement, au XVII ${ }^{\mathrm{e}}$ siècle, la Logique de Port Royal d'Arnauld et Nicolle, traitait par la logique, la détermination des termes, sans avoir su, il est vrai, accompagner ses propos d'une formalisation comparable à l'algèbre de Boole et encore moins au calcul frégéen des prédicats. Des problèmes comme le traitement sémantique des anaphores dans des énoncés analogues aux "donkey sentences » (ex.: Lorsque Pedro possède un âne, il le bat) ou aux phrases sataniques (ex.: Satan est le seul à n'avoir pitié que de soi-même) montrent bien que les analyses et représentations formulées dans le seul calcul des prédicats sont nettement insuffisantes. Il en est de même des ambiguités référentielles discursives comme dans un échange extrait d'une pièce de théâtre de boulevard: J'aime ma femme, dit le mari; Moi aussi, répond l'amant, marié de son côté, puisque moi aussi peut faire référence soit à « la femme du mari », soit à « la femme » de l'amant.

La logique moderne ne permet ni de composer des prédicats entre eux pour former des prédicats complexes (exemples: 'se-lever $(\mathrm{x})$ ', 'posséder-etbattre $(\mathrm{x}, \mathrm{y})$ ' ou encore 'aimer-ce-quiest-propre-à-soi (x)' dans aimer sa (propre) ferme), ni de composer des prédicats avec des opérateurs non prédicatifs comme des prépositions, des préfixes préverbaux, (souvent reliés à des prépositions) ou des marqueurs de réflexivité (exemples: 'sortir-de'/ 'donner-à'/ 'surmonter' / 'monter sur' / 'ex-filtrer' et 'infiltrer/ 's'entourer-de' / 's'entre-tuer' .... ${ }^{40}$,

La Logique Combinatoire (LC), introduite au $\mathrm{XX}^{\mathrm{e}}$ siècle, a été essentiellement développée par H. B. Curry et ses disciples. ${ }^{41}$ Cette logique donne la possibilité de formaliser les opérations formelles de substitution dans les déductions, en évitant les am-

\footnotetext{
40 Pour des solutions apportées à ces problèmes, voir J.P. Desclés et al. Vol. II, 2016, o.c., pp. 356-418.

41 H. B. Curry and R. Feys, Combinatory Logic, Vol. I, 1958; J. R. Hindl ey and J. P. Seldin, Lamda-calculus and Combinators, an introduction, Cambridge: Cambridge University Press, 2008; voir aussi J.-P. Desclés, G. Guibert et B. Sausay, vol. I, ,o.c., 2016.
} 
biguïtés usuelles et les pièges des «variables liées » pourtant utilisées dans les discours des mathématiques, la logique moderne et le $\lambda$-calcul de Church (1941), ce qui oblige souvent à devoir " renommer les variables » dans certaines substitutions pour éviter les incohérences sémantiques. Étant un formalisme "sans variables liées ", la LC analyse l'émergence des expressions paradoxales, formulables dans une langue naturelle; de telles expressions ont été déclarées "sans signification » par B. Russell, et par là, éliminées du champ de la logique, ce que n'accepte pas H.B. Curry. La LC permet d'étudier l'émergence des paradoxes et de proposer des solutions aux problèmes posés par les compositions de prédicats plus élémentaires avec des opérateurs que les langues expriment.

Remarque: La logique moderne représente le schéma prédicatif binaire 'Luc aimer Marie' par 'aimer ${ }_{2}(<$ Luc, Marie $>)$ ', ce qui revient à appliquer le prédicat binaire directement au couple $<$ Luc, Marie $>$, sans distinguer les différents rôles syntaxiques et sémantiques des arguments; la LC analyse la constitution de la proposition 'Luc aimer Marie' au moyen d'une double opération de prédication, représentée soit par '(aimer ${ }_{2}$ (Marie)) Luc', soit par '( aimer $_{2}$ (Luc)) Marie', ce qui permet de mieux examiner le problème linguistique des rôles syntaxiques du « sujet » et du «complément », bien qu'il y ait une correspondance entre les deux représentations, selon le principe de Schönfinkel-Curry, essentiel aux représentations applicatives de la LC, puisque cette dernière travaille essentiellement avec des opérateurs unaires (de différents types fonctionnels), " équivalents » pour certains d'entre eux à des opérateurs n-aires, selon le principe de Schönfinkel-Curry.

Avec le formalisme de la LC, il devient possible de tenir compte des variations sémantiques importantes apportées par les différentes diathèses (ergatives, transitives, moyennes, réflexives, passives et médio-passives), par les constructions impersonnelles, par les opérations dans l'opposition thème/ rhème et par les opérations de détermination de ses différents constituants. La LC permet également de représenter les différentes opérations énonciatives de prise en charge par un énonciateur d'une relation prédicative, c'est-à-dire un dictum (ou une lexis). Toutes ces opérations sont des résultats de compositions de divers types d'opérateurs (catégorisés selon la théorie des types fonctionnels de Church), elles deviennent des constituants essentiels à l'activité de langage. La LC permet également d'associer, par des déductions formelles explicites, les expressions linguistiques d'une langue à leurs interprétations représentées à l'aide de schèmes instanciés formulés dans le cadre de la LC.

Remarque: Les exemples qui viennent d'être mentionnés, sous forme de déductions entre expressions et interprétations, sont exposés dans nos publications. 42

Dans notre approche philosophique

42 Voir J.-P. Desclés et al., vol. II, 2016, o.c. pp. 357-450, 451-496, 497-531. 
de la logique, la Logique Combinatoire, dénommée ainsi par H.B. Curry, doit être pensée comme une logique d'opérateurs quelconques, composables (ou décomposables) et transformables par des processus opératoires intrinsèques, ce qui veut dire indépendants des domaines d'action des opérateurs composés ou transformés; ces processus opératoires intrinsèques de composition et de transformation sont effectués au moyen d'opérateurs abstraits, appelés "combinateurs »; chaque combinateur a son action opératoire qui est précisée par une règle d'introduction et par une règle d'élimination, formulées selon le style de la "Déduction Naturelle » de Gentzen. Un nombre très restreint de combinateurs élémentaires permet d'engendrer récursivement tous les combinateurs qui effectuent outes les formes de composition (par exemple, des intrications en parallèle d'opérateurs) et de transformation (par exemple, en dupliquant un argument d'un opérateur binaire), en construisant ainsi des opérateurs complexes. Nous n'avons pas la place de présenter ici les éléments techniques de la LC avec des exemples illustratifs de traitement de problèmes linguistiques évoqués; nous renvoyons à nos publications.

La LC typée (par les types fonctionnels de Church) comprend différents types d'opérateurs et d'opérandes absolus (qui ne sont jamais des opérateurs) spécifiques d'une part, à la syn- taxe et d'autre part, à la sémantique d'une langue. Par des calculs déductifs, un énoncé peut être relié à sa représentation interprétative, dans laquelle des relations de repérage jouent le rôle de « coordonnées énonciatives " d'une relation prédicative aspectualisée et modalisée. A titre d'exemple, prenons deux énoncés élémentaires avec leurs représentations énonciatives:

(a) Hier, je dormais

(a') PROC J0 $_{0}$ EGO-DIT

$\left(\left(\left[\right.\right.\right.$ hier $_{\mathrm{O} 2}\left(\right.$ PROC $_{\mathrm{J} 1}\left(\right.$ dormir $\left.\left.\left.\left.\mathrm{T}^{1}\right)\right)\right]\right)$

$\&\left(\left[\mathrm{O}_{2}<\mathrm{J}_{0}\right] \&\left[\mathrm{~J}_{1} \subset \mathrm{O}_{2}\right] \&\left[\mathrm{~d}\left(\mathrm{~J}_{1}\right)<\mathrm{d}\left(\mathrm{J}_{0}\right)\right]\right)$ $\left.\&\left(\left[\mathrm{~T}^{1}=\mathrm{EGO}\right]\right)\right)$

(b) Aujourd'hui, tu me vois

(b') PROC J0 o EGO-DIT

( ([aujourd'hui O2 $\left(\right.$ PROC $_{\mathrm{J} 1}\left(\right.$ voir $\left.\left.\left.\left.^{2} \mathrm{~T}^{1}\right)\right)\right)\right]$ ) $\&\left(\left[\mathrm{O}_{2} \supset \mathrm{J}_{0}\right] \&\left[\mathrm{~J}_{1} \subset \mathrm{O}_{2}\right] \&\left[\mathrm{~d}\left(\mathrm{~J}_{1}\right)=\mathrm{d}\left(\mathrm{J}_{0}\right)\right]\right)$ $\left.\&\left(\left[\mathrm{~T}^{1} \neq \mathrm{EGO}\right] \&\left[\mathrm{~T}^{2}=\mathrm{EGO}\right]\right)\right)$

Dans ces analyses, les unités grammaticales sont les traces linguistiques d'opérateurs aspectuels et de relations de repérage:

(a") hier $=$ trace des deux relations

$$
\begin{aligned}
j e= & {\left[\mathrm{O}_{2}<\mathrm{J}_{0}\right] \text { et }\left[\mathrm{J}_{1} \subset \mathrm{O}_{2}\right] } \\
\text {-ais }= & \text { trace de }\left[\mathrm{T}^{1}=\mathrm{EGO}\right] \\
& {\left[\mathrm{d}\left(\mathrm{J}_{1}\right)<\mathrm{d}\left(\mathrm{J}_{0}\right)\right] \&\left[\mathrm{~T}^{1}=\mathrm{EGO}\right] . }
\end{aligned}
$$

(b") aujourd'hui $=$ trace des relations

$$
\begin{aligned}
& {\left[\begin{array}{ll}
\left.\mathrm{O}_{2} \supset \mathrm{J}_{0}\right] \text { et }\left[\mathrm{J}_{1} \subset \mathrm{O}_{2}\right.
\end{array}\right]} \\
& t u=\operatorname{trace} d e\left[\mathrm{~T}^{1} \neq \mathrm{EGO}\right] ; \\
& m e=\operatorname{trace} \text { de }\left[\mathrm{T}^{2}=\mathrm{EGO}\right] \\
& -s=\operatorname{trace} \text { de l'opérateur PROC } \mathrm{I}_{\mathrm{J} 1} \text { et de } \\
& \quad\left[\mathrm{d}\left(\mathrm{J}_{1}\right)=\mathrm{d}\left(\mathrm{J}_{0}\right)\right] \&\left[\mathrm{~T}^{1} \neq \mathrm{EGO}\right] .
\end{aligned}
$$


Les relations et opérateurs constitutifs des coordonnées énonciatives sont composées au moyen de combinateurs de façon à construire des représentations exprimées par leurs traces linguistiques sous la forme d'unités synthétiques. Nous ne précisons pas ici ces calculs effectués à l'aide de combinateurs.

Par une démarche onomasiologique de synthèse intégrative, le schème énonciatif qui emboîte la relation prédicative aspectualisée, par exemple 'ASP ${ }_{\mathrm{I}}\left(\mathrm{P}_{2} \mathrm{~T}^{2} \mathrm{~T}^{1}\right)$ ', est reliée, par une déduction naturelle "à la Gentzen », à son expression morphosyntaxique puis à son expression dans une séquence syntagmatique concaténée. Cette démarche s'effectue en plusieurs étapes par l'introduction de combinateurs; à une certaine étape de la déduction, sont introduits deux quantificateurs existentiels (qui éliminent, dans la représentation, les noms $\mathrm{J}_{0}, \mathrm{~J}_{1}, \mathrm{O}_{1}, \mathrm{O}_{2}$ des intervalles temporels qualitatifs), ce qui permet de dégager un opérateur aspectuotemporel complexe 'asp-tps' qui a pour opérande la relation prédicative entière, d'où l'expression 'asp-tps $\left(\mathrm{P}_{2} \mathrm{~T}^{2} \mathrm{~T}^{1}\right)$ '; cet opérateur 'asp-tps' peut se composer ensuite avec le prédicat $\mathrm{P}_{2}$, pour produire une unité morphosyntaxique synthétique qui peut alors être inséré dans une séquence syntagmatique, concaténée par '+'. Les principales étapes de la synthèse intégrative précédente 43 sont ainsi résumées comme suit:

$$
\begin{aligned}
\text { PROC }_{\mathrm{J} 0}\left({ }_{1}\right. \text { EGO-DIT } \\
\quad \\
\left.\left.\quad{ }_{2} \&\left({ }_{3} \mathrm{ASP}_{\mathrm{I}}\left(\mathrm{P}_{2} \mathrm{~T}^{2} \mathrm{~T}^{1}\right)_{3}\right)\left(\in \mathrm{J}_{0} \mathrm{I}\right)_{2}\right)_{1}\right) \\
->\operatorname{asp-tps}\left(\mathrm{P}_{2} \mathrm{~T}^{2} \mathrm{~T}^{1}\right) \\
->\left(\text { morph-asp-tps } \mathrm{P}_{2}\right) \mathrm{T}^{2} \mathrm{~T}^{1} \\
->\mathrm{S}^{1}+(\mathrm{V}+\text {-morph-asp-tps })+\mathrm{O}^{2}
\end{aligned}
$$

Dans une démarche sémasiologique de décomposition analytique, le calcul part d'une expression concaténée, une instance du schéma syntagmatique:

$$
\mathrm{S}^{1}+(\mathrm{V}+- \text { morph-asp-tps })+\mathrm{O}^{2}
$$

Par une analyse effectuée dans le cadre des grammaires catégorielles, le suffixe grammatical verbal '-morph-asptps' est analysé comme un opérateur qui a pour argument le radical verbal ' $\mathrm{V}$ '; cet opérateur grammatical est ensuite identifié à un opérateur complexe, qui se décompose en un opérateur aspectuel plus abstrait, accompagné d'une relation temporelle, ce qui aboutit finalement à reconstruire une représentation interprétative de l'énoncé de départ.

En poussant formellement jusqu'au bout les analyses de la Grammaire d'opérateurs de Z. Harris (1976, 1983), nous avons montré, avec l'outil de la LC, que l'analyse harissienne pourrait utiliser adéquatement des combinateurs de la LC, pour construire une forme interprétative, ce que ne fait pas

\footnotetext{
${ }^{43}$ Les calculs détaillés de telles déductions sont présentés dans nos diverses publications; voir en particulier, Desclés et al., vol. II, 2016, o.c. pp. 504-528.
} 
Z. Harris qui ne détaille pas la formalisation des opérateurs de sa grammaire, en restant dans une métalangue de gloses interprétatives, puisque, pour Harris, " la métalangue est dans la langue $» .44$

Nous venons d'indiquer très brièvement comment il était possible de relier par des déductions effectuées dans le cadre de la LC, des marqueurs grammaticaux à leurs interprétations énonciatives représentées par des expressions applicatives, qui étaient le résultat de compositions d'opérateurs plus élémentaires. Nous allons maintenant montrer comment un prédicat verbal peut être relié à sa signification. Remarquons qu'une unité prédicative comme 'sortir de' est polysémique comme, du reste, la majorité des prédicats verbaux. Prenons quelques exemples de constructions syntaxiques de 'sortir de', où les actants n'ont pas le même rôle sémantique:

(a) La voiture sort du garage.

[mouvement cinématique sans agent]

(b) Luc sort la voiture du garage

[mouvement dynamique avec agent];

(c) Luc sort la voiture du garage avec un tracteur.

[mouvement dynamique avec agent et instrument]

(d) Luc fait sortir la voiture du garage par son chauffeur.

[mouvement dynamique factitif effectué par un agent sous le contrôle d'un autre agent]

(e) Les fleurs sortent de terre au printemps.

[changement d'états sans agent]

L'interprétation de (a) est donnée à l'aide d'un schème cinématique (a') construit avec la primitive relationnelle $\mathrm{du}$ mouvement $\mathrm{MOUVT}_{\mathrm{F}}$ actualisé sur l'intervalle fermé ' $\mathrm{F}$ ' de la transition entreles deux ouverts $\mathrm{O}_{1}$ et $\mathrm{O}_{2}$ où sont actualisées deux situations statiques, dans lesquelles un objet 'y' est repéré, à l'aide de ' $\epsilon$ ', par rapport à l'intérieur, puis à l'extérieur d'un même lieu Loc(z) (avec $\mathrm{y}=$ la voiture, $\mathrm{z}=$ le garage):

$\left(\mathrm{a}^{\prime}\right)\left[\operatorname{SIT}^{1} \mathrm{O} 1[\mathrm{y}, \mathrm{z}] \mathrm{MOUVT}_{\mathrm{F}} \operatorname{SIT}^{2} \mathrm{O} 2[\mathrm{y}, \mathrm{z}]\right.$ ] où $\left[\mathrm{O}_{1}<\mathrm{O}_{2}\right]$ avec:

$$
\begin{aligned}
& \operatorname{SIT}^{1} \mathrm{O} 1[\mathrm{y}, \mathrm{z}]=[\mathrm{y} \in \mathrm{O} 1(\operatorname{Int}(\operatorname{Loc}(\mathrm{z}))] \\
& \operatorname{SIT}_{\mathrm{O} 2}^{2}[\mathrm{y}, \mathrm{z}]=[\mathrm{y} \underline{\underline{\epsilon}} \mathbf{\mathrm { O } 2}(\underline{\underline{\operatorname{Ext}}}(\operatorname{Loc}(\mathrm{z}))]
\end{aligned}
$$

Le schème interprétatif de (b) est plus complexe puisqu'il s'agit d'un schème dynamique ( $\mathrm{b}$ ') avec un agent ' $\mathrm{x}$ ' qui contrôle l'effectuation du mouvement d'un objet mobile ' $y$ ' (avec: $\mathrm{x}=\mathrm{Luc}$; $\mathrm{y}=\mathrm{la}$ voiture; $\mathrm{z}=$ le garage):

(b') $\left[x \operatorname{CONTR}_{\mathrm{F}}\left({ }_{1} \times\right.\right.$ FAIRE $_{\mathrm{F}}$ $\left.\left.\left({ }_{2}\left[\operatorname{SIT}^{1}{ }_{\mathrm{O} 1}[\mathrm{y}, \mathrm{z}] \operatorname{MOUVT}_{\mathrm{F}} \operatorname{SIT}^{2} \mathrm{O} 2[\mathrm{y}, \mathrm{z}]\right]_{2}\right)_{1}\right)\right]$ où $\left[\mathrm{O}_{1}<\mathrm{O}_{2}\right]$ avec:

$$
\operatorname{SIT}^{1}{ }_{\mathrm{O} 1}[\mathrm{y}, \mathrm{z}]=\left[\mathrm{y} \in \mathrm{O}_{\mathrm{O}}(\underline{\operatorname{Int}}(\operatorname{Loc}(\mathrm{z}))]\right.
$$

\footnotetext{
44 J.-P. Desclés, «Les mathématiques de la grammaire d'opérateurs de Zellig Harris », in C. Martinot et al., Perspectives harissiennes, Cellule de recherche linguistique, Paris, 2016, pp. 83-105; voir en particulier la déduction dans l'annexe, pp. $104-105$.
} 


$$
\operatorname{SIT}^{2} \mathrm{O}_{2}[\mathrm{y}, \mathrm{z}]=\left[\mathrm{y} \in \mathrm{O}_{2}(\underline{\operatorname{Ext}}(\operatorname{Loc}(\mathrm{z}))]\right.
$$

Ce schème ( $\left.b^{\prime}\right)$ est construit en plaçant le schème $\left(a^{\prime}\right)$ sous le contrôle de l'agent ' $x$ ', le mouvement de ' $y$ ' étant effectué par l'agent lui-même ' $x$ '.

Le schème $\left(c^{\prime}\right)$ de $(c)$ est obtenu à partir de (b'), en substituant un instrument $(\mathrm{u}=$ un tracteur) à l'agent $\mathrm{x}$ qui effectue le mouvement. Le schème factitif (d') de (d) introduit le contrôle d'un premier agent sur un second agent qui, lui, est directement chargé de l'effectuation du mouvement d'un objet mobile. Le schème cinématique ( $\left.\mathrm{e}^{\prime}\right)$ de (e) représente un changement d'états d'une même entité, sans agent exprimé.

Tous ces schèmes sont des représentations de relations simples ou plus complexes construites avec des emboîtements de relations dans une relation plus globale.

Une entité est agentive lorsqu'elle contrôle l'action; le contrôle signifie, comme nous l'avons déjà dit, la mise en œuvre de la capacité de déclencher ou d'interrompre d'une action, qui peut être effectuée par un intermédiaire (un instrument) ou parfois par l'agent lui-même. Cette caractérisation de l'agent est donc différente des définitions ontologiques (sous la forme de traits comme '+ animé', '+ humain'...) présentées usuellement par la littérature linguistique.
Remarque: La notion de « contrôle » est utilisée par des linguistes comme B. Comrie, C. Hagège, G. Lazard, sans que les propriétés de déclenchement et d'interruption d'une action aient été dégagées. 45

Une entité qui est usuellement catégorisée, du point de vue de la nature, comme une "force de la nature " non agentive, peut cependant être présentée dans certaines énonciations comme jouant le rôle d'un agent intentionnel qui ainsi contrôle une action orientée vers un but déterminé (exemple: Pour se venger des marins qui viennent, chaque année, fouiller, avec leurs filets, ses entrailles, la mer se décida brusquement à envahir les ports et à déclencher des tempêtes qui finiront par avaler toutes les embarcations de ces maudits pêcheurs). Une entité qui, dans le discours, est présentée comme exerçant une capacité de contrôle, acquiert le rôle (discursif) d'un agent avec une visée téléonomique, contrairement à la plupart de ses emplois prototypiques (exemple: Décidemment, cette pierre m'en veut car elle s'arrange pour me faire trébucher presque tous les soirs quand je viens rejoindre ma famille.). Inversement, un être humain ou un être animé, qui sont des entités amenées à exercer assez naturellement des rôles d'agents, peut perdre sa capacité habituelle de contrôle; ainsi, on peut comparer d'un côté, Luc est tombé sans le vouloir qui indique que Luc n’a pas exercé sa capac-

45 Voir Desclés et al., vol. II, 2016, o. c., pp. 420-435. 
ité d'un agent prototypique, avec, d'un autre côté, l'énoncé Pour se faire remarquer de tous ses camarades, le jeune collégien Salvador Dali est tombé, plusieurs jours de suite, juste avant de pénétrer dans la cour de récréation.

Dans cet article, nous avons brièvement argumenté qu'une langue pouvait être appréhendée sous la forme d'un système sémiotique applicatif, composé d'opérateurs de différents types fonctionnels (ou catégories) qui, en s'appliquant à leurs opérandes, construisent, en plusieurs étapes, les unités sémiotiques que sont les phrases et les énoncés. Les grammaires catégorielles (étendues) ${ }^{46}$ réalisent cette première étape qui aboutit à associer et à construire des expressions applicatives, composées d'opérateurs morphosyntaxiques, des préalables aux constructions sémantiques ultérieures de représentations sémantiques, c'est-à-dire des instanciations de schèmes interprétatifs spécifiques à chaque langue. Le schème qui représente la signification d'une unité verbale ou prépositionnelle est le résultat d'une composition de primitives dont certaines ont un ancrage cognitif dans les domaines de la perception et de l'action. Si les représentations interprétatives sont "mentales", elles ne sont pas observables mais les traces lin- guistiques qu'elles laissent dans les organisations des énoncés le sont et donnent la possibilité aux linguistes de les " reconstruire ", à condition toutefois de préciser dans le détail toutes les différentes opérations de changement de représentations.

En utilisant systématiquement les systèmes applicatifs, la philosophie du langage et la linguistique théorique se dotent de moyens techniques pour étudier le rôle de la formalisation applicative, avec ses opérateurs et opérandes de différents types, dans l'approche de la compréhension de la nature profonde du langage humain, appréhendé à partie des systèmes des langues. Pour cela, la linguistique théorique doit montrer comment: $1^{\circ}$ ) les opérateurs linguistiques peuvent se composer entre eux afin de construire des opérateurs plus complexes (certains d'entre eux étant directement exprimés par des unités linguistiques directement composées entre elles), et $2^{\circ}$ ) interpréter ces opérateurs complexes par des schèmes, qui sont également des représentations applicatives. Les représentations sémantiques ne doivent pas se réduire à de simples « vecteurs » de traits classificatoires (pseudo-) booléens ${ }^{47}$, comme c'était le cas dans l'approche structurale de la sémantique. La sémantique cogni-

\footnotetext{
46 Voir, par exemple, I. Biskri et J.-P. Desclés, « Applicative and Combinatory Categorial Grammar (from syntax to functional semantics) », in R. Mitkov et N. Nikolov (eds), Recent Advances in Natural Language Processing, John Benjamins, 1997, pp. 71-84; voir aussi J.-P. Desclés et al. , vol. II, 2016, o.c., pp. 305-355.

47 Etant donné un trait ' $A$ ', la linguistique structurale (comme dans l'approche de E. Coseriu) envisage le trait marqué ' $+A^{\prime}$ ' le trait non marqué ' $-A$ ', le neutre c'est-à-dire le trait ' $+A$ ou $-A$ ', et le trait ' $n i+A$, ni $-A$ '.
} 
tive a cherché dépasser le cadre strictement structuraliste. C'est le cas du parcours scientifique de B. Pottier puisque, au début de sa carrière, il recourait à des analyses sémantiques structurales détaillées, mais il a su complexifier sa démarche avec des représentations sémantiques plus structurées, comme les schèmes analytiques, dont le trimorphe déjà évoqué plus haut.

Avec les compositions, qui ne sont pas toujours simplement fonctionnelles, et les transformations d'opérateurs de différents types, qu'effectuent les combinateurs de la LC, il devient possible de répondre aux besoins opératoires des constructions des significations structurées sous la forme de relations de relations parfois intriquées (car portant sur des arguments identiques), et cela de façon peut-être plus adéquate que le $\lambda$-calcul de Church, pourtant largement utilisé dans des approches de la sémantique formelle. ${ }^{48}$ En effet, pour exprimer les opérateurs, le $\lambda$-calcul doit utiliser des "variables liées », ce qui entraîne de nombreux renommages de variables au cours des compositions et des applications des expressions-opérateurs à des opérandes, avec parfois la création d'erreurs, assez difficiles à détecter et parfois à l'origine de "bugs » dans les programmes informatiques. Or, la LC, et c'est là une de ses grandes qualités, est une logique "sans variables liées » qui, grâce à ses combinateurs, gère facilement la portée applicative des opérateurs sur leurs opérandes, situées même à distance. 49

Dans notre approche de la sémantique à l'aide de schèmes interprétatifs engendrés par des compositions de primitives sémantico-cognitives, les représentations des significations ne sont pas universelles et indépendantes des langues. Cela n'est pas le cas des représentations applicatives de la Grammaire Applicative Universelle de S. K. Shaumyan ${ }^{50}$ qui a cherché, également avec les outils des grammaires catégorielles et de la LC, à construire un "langage génotype » universel (une forme de "langage de la pensée ») dont les langues phénotypes, avec leurs particularités morphosyntaxiques et phoniques, seraient les projections. Nous ne souscrivons pas à cette vue trop universaliste puisque, selon nous, chaque langue organise ses propres réseaux (en particulier polysémiques) de schèmes, ce qui indique que la pensée est en grande partie dépendante de la langue utilisée pour échanger avec autrui d'une communauté linguistique, éventuellement avec soi-même.51

\footnotetext{
48 C'est le cas, par exemple, dans les analyses entreprises par R. Montague (1974) et de tous ceux qui s'en inspirent directement.

49 Voir Desclés et al., vol. I, 2016, o.c., pp. 145-174.

${ }^{50}$ Voir, par exemple, S.K. Shaumyan, A Semiotic Theory of Language, Bloomington, Indiana University Press, 1987.

51 Nous adoptons une approche anti-anti-relativiste; voir, à ce propos, J.-P. Desclés, « Les représentations cognitives du langage sont-elles universelles? », in Essais sur le langage, logique et sens commun, Fribourg, Suisse, Editions Universitaires, 1998 , pp. 53-81.
} 


\section{Principe de la compilation général- isée}

L'analyse des opérations énonciatives et des représentations sémantiques des lexèmes, avec le formalisme applicatif de la LC, nous a amené à proposer l'architecture linguistique et computationnelle du modèle appelé GRammaire Applicative, Cognitive et Enonciative (GRACE); ce modèle est complexifie l'architecture antérieure de la Grammaire Applicative et Cognitive et se différencie, sur de plusieurs points, de la Grammaire Applicative Universelle de S. K. Shaumyan. Les changements de représentations entre niveaux d'analyse dans ces architectures polystratales reposent sur l'hypothèse caractérisée par une forme de " compilation généralisée » liée à l'activité de langage qui associe des expressions linguistiques avec leurs représentations interprétatives, non pas directement mais en engendrant des représentations intermédiaires. ${ }^{52}$ Ce principe s'inspire de l'informatique qui s'est développée grâce à la compilation des langages, mise en évidence par J. Backus, le créateur du premier langage de programmation de haut niveau FORTRAN; les programmes écrits dans ce langage de programmation sont assez proches des formulations algébriques et logiques des mathématiciens, ils sont indépendants du langage machine, composé de séquences d'instructions détaillées directement exécutables par les organes de calcul d'une machine informatique et de sa mémoire où sont localisés physiquement les données et les résultats intermédiaires. Le principe de la compilation, souvent mal compris des non informaticiens, se résume ainsi: si l'on veut d'une part, exprimer des algorithmes sous la forme de programmes écrits dans un langage symbolique structuré dit "de haut niveau » (un langage de programmation) et d'autre part, faire exécuter ces programmes par des organismes matériels (électroniques, éventuellement quantiques ou même neurobiologiques) en mémorisant certaines associations déjà calculées afin de les réutiliser directement dans l'exécution d'autres programmes, alors il ne faut pas chercher à associer directement les programmes à leurs représentations exécutables par les organes physiques d'une machine informatique; il faut plutôt procéder à des changements de représentations intermédiaires à différents niveaux articulés entre eux par des processus opératoires de changement des représentations. Pour cela, étant donné un langage de programmation $\mathrm{L}$, choisi pour écrire les programmes que l'on souhaite voir

\footnotetext{
52 J. P. Desclés, "Cognition, compilation et langage ", in Chazal et Terasse (eds), Philosophie du langage et informatique, Paris, Hermès, 1996, pp. 103-145; voir aussi Desclés et al., vol. II, 2016, o.c.
} 
être exécutés par une machine $M$, il convient d'écrire un autre programme très général $\mathrm{KL}$, appelé " compilateur,$\sqrt{53}$, ce programme qui est spécifique à la machine $M$ et au langage $L$, est exécutable (on dit aussi implanté) sur la machine $\mathrm{M}$; le compilateur KL doit gérer, pour n'importe quel programme écrit dans le langage de programmation $\mathrm{L}$, tous les changements de représentations intermédiaires qui s'imposent pour se rapprocher du langage machine et ainsi relier automatiquement, d'un côté, toutes les représentations externes formulées selon la syntaxe du langage $\mathrm{L}$ (de haut niveau) avec, d'un autre côté, les représentations exprimées dans le langage machine interne de M. C'est grâce au compilateur KL qu'un programmeur peut écrire, en respectant scrupuleusement la syntaxe du langage de programmation L, un programme PL qui formule des algorithmes, sans qu'il ait besoin de gérer toutes les phases intermédiaires des changements de représentation du programme PL, avant l'exécution matérielle du programme PL, tous ces changements étant délégués au compilateur KL; ce programme KL a, en outre, la tâche de détecter et de signaler les éventuelles incorrections syntaxiques et les anomalies logiques détectées dans l'écriture du programme PL avant même son exécution par la machine $\mathrm{M}$. La relation compilée entre l'expression externe du programme $\mathrm{PL}$ dans le langage $\mathrm{L}$ et sa représentation interne dans le langage machine de $\mathrm{M}$ peut être mémorisée dans $M$ et directement réutilisée lorsque le programme s'applique à de nouvelles données. Le langage HASKELL est un langage de programmation, dite fonctionnelle, parce qu'il possède une syntaxe (et une sémantique dénotationnelle) qui est assez analogue à la syntaxe des expressions applicatives de la logique combinatoire (LC) de Curry. La compilation de HASKELL permet d'écrire dans ce langage fonctionnel des programmes qui sont ensuite exécutés facilement sur des machines informatiques.

Dans les années 1960-1970, la traduction automatique s'est inspirée de la compilation en informatique, avec ce qui est maintenant appelé " le triangle de Vauquois ", en effectuant la traduction du texte $\mathrm{T}$ de la langue source par la construction de plusieurs niveaux intermédiaires avec leurs représentations: identification des unités, analyse et découpage morphologique puis syntaxique et, enfin, représentation sémantique RS(T) du texte T pour ensuite entreprendre la traduction en engendrant, à partir de $\mathrm{RS}(\mathrm{T})$, son découpage syntaxique et morphologique dans la langue cible.

En dépassant la stricte technique informatique, la réflexion philosophique menée à propos de la compilation dé-

\footnotetext{
53 Voir, G. Berry, L'Hyperpuissance de l'informatique. Algorithmes, données, machines, réseaux, Odile Jacob, Paris, 2017 pp. $448-451$.
} 
gage l'idée générale: une pensée complexe, qui s'exprime sous la forme d'une succession organisée d'opérateurs complexes de différents types appliqués à des opérandes (en fait un programme fonctionnel), peut être implantée dans des organes matériels ayant une toute autre organisation structurelle, en procédant à divers changements de représentation.

Pour les recherches menées sur la sémantique des langues, l'objectif est clair. Il s'agit de s'inspirer du principe de la compilation généralisée, puis de proposer des architectures cognitives et computationnelles de l'activité de langage 54 en décrivant des formes de compilation par changements de représentations entre différents niveaux intermédiaires (phonoprosodiques, morphologiques et syntaxiques, sémantiques et énonciatifs), afin de réaliser des simulations expérimentales effectives qui permettent de déboucher ultérieurement sur des applications précises (traduction automatique de qualité, fouille sémantique de textes, génération automatique de discours, fiches de synthèse de plusieurs documents...). Dans ce genre de modélisation, l'énoncé (puis, de façon plus générale et plus complexe, un discours) peut être conçu comme étant une sorte de programme fonctionnel où des unités linguistiques peuvent se composer et s'appliquer à des opérandes également linguistiques; par un processus général de compilation, le programme-énoncé est transformé, à la suite de différents changements intermédiaires, en représentations sémantico-cognitives; ces dernières sont à leur tour transformées, vraisemblablement en plusieurs étapes intermédiaires, en des instructions implantées sur des réseaux neuronaux et exécutées, les unes après les autres ou en parallèle. Ces deux processus de compilation, l'un reliant expression sémiotique externe et interprétation sémantique interne, l'autre reliant représentation sémantique et son implantation sur les réseaux neuronaux du cerveau, permettraient sans doute de mieux saisir et d'étudier comment fonctionne l'activité de langage.

Le premier processus de compilation, essentiellement cognitif, devient un thème central pour la recherche sémantique en linguistique, en logique et en psychologie cognitive. Dans ce cas, il ne s'agit donc plus de décrire uniquement les différents niveaux de représentation mais aussi de décrire le processus de changement des représentations entre les expressions externes et les représentations interprétatives internes; cela revient à décrire une sorte de compilateur, associé à une langue, chargé d'expliquer comment les énon-

\footnotetext{
${ }^{54}$ Plusieurs architectures polystratales ont été développées par la linguistique, par exemple: le modèle «sens-texte » de I. Mel’čuk; le modèle de la Grammaire Applicative Universelle de S. K. Shaumyan; les modèles de la Grammaire Applicative et Cognitive et de la Grammaire Applicative, Cognitive et Énonciative.
} 
cés sont compris et produits.

Le second processus de compilation relève plus directement des recherches menées par les neurosciences; ces dernières devraient maintenant s'intéresser à décrire non plus les seules associations directes entre la reconnaissance de signaux externes (identification d'un mot par exemple) et le déclenchement d'un certain état du système neuronal du cerveau, mais aussi tenter d'expliquer comment les significations des unités linguistiques isolées et organisées dans des unités plus vastes (comme les énoncés) sont effectivement implantées sur les structures neuronales du cerveau, traitées et composées par elles. Malgré des résultats fascinants et très intéressants, les travaux menés actuellement par les neuroscience, sont encore loin de proposer des modélisations explicatives qui soient compatibles avec les recherches actuelles menées par la sémantique cognitive; ces travaux doivent chercher à expliciter comment la signification des unités lexicales et grammaticales d'une langue sont implémentées et traitées par les organes neuronaux; plus généralement, il s'agit d'expliquer comment le cerveau arrive à construire puis à mémoriser le contenu d'un énoncé, pour ensuite entreprendre de produire des paraphrases, déclencher certaines inférences à partir de la compréhension d'un énoncé, associer plus ou moins fidèlement des énoncés linguistiques et des représentations iconiques et figuratives ou des images. Comme l'a montré D. Marr ${ }^{55}$, la vision procède justement par des changements de représentations en passant des représentations d'un espace 2D, captées par la rétine, à des représentations internes d'un espace 3D. Pourquoi le traitement de la sémantique des langues par le cerveau ne ferait-il pas appel, lui aussi, à des changements de représentations, selon le principe de la compilation?

Le siècle dernier prévoyait que l'avenir de l'Intelligence Artificielle (IA) devrait se focaliser sur des représentations de connaissances, de plus en plus déclaratives et hiérarchisées les unes par rapport aux autres, en déterminant également des métaconnaissances, conçues surtout pour décrire des stratégies intelligentes orientées vers la réalisation d'un but précis ${ }^{56}$; ces connaissances et métaconnaissances, une fois transmises aux machines informatiques, devaient guider les calculs impliquées par un nombre important et diversifié de tâches réputées intelligentes, comme battre les grands Maîtres des échecs ou du jeu de Go, ou, problème plus difficile, apporter des solutions générales à tous les problèmes

\footnotetext{
${ }^{55}$ D. Marr, Vision, Freeman and Company, New York, 1982

${ }^{56}$ Voir, par exemple, Th. Winograd et F. Flores, L'intelligence artificielle en question, Paris, Presses Universitaires de France, 1986; G. Sabah, L'intelligence artificielle et le langage, processus de compréhension, Paris, Herrmès, 1989; J. Pitrat, Métaconnaissances, futur de l'intelligence artificielle, Paris, Hermès, 1990.
} 
que la compréhension et la production automatiques d'énoncés, de discours et de textes, soulèvent. On ne peut que constater que l'IA actuelle s'est considérablement détournée de cette voie qui travaillait avec des représentations plus ou moins déclaratives de connaissances et de métaconnaissances, pour se focaliser sur des simulations de prises de décision, effectuées à la suite d'apprentissages fondés sur des statistiques extraites de très vastes bases de données (big data), souvent annotées, avec des apprentissages " profonds» (deep learning) (souvent sans annotations préalables). ${ }^{57}$ L'IA semble maintenant développer surtout des technologies socialement réclamées pour répondre à des applications pratiques et bien ciblées mais parfois aux horizons limités et pas toujours facilement généralisables. Grâce aux performances des ordinateurs actuels capables d'effectuer très rapidement des calculs de plus en plus complexes et à stocker un volume de plus en plus important de données, des programmes ont réussi, à la suite d'apprentissages exercés sur de très vastes bases de jeux enregistrés, à battre des grands maîtres des jeux d'échecs puis de Go. A partir d'un apprentissage basé sur un grand nombre de corpus de textes traduits, la traduction automatique et la fouille textuelle obtiennent des résultats relativement satisfaisants, du moins lorsqu'il s'agit de traiter de domaines bien circonscrits. Ces succès incontestables soulèvent cependant de nouvelles questions épistémologiques non négligeables, tout particulièrement en ce qui concerne la nature du langage. Mentionnons quelques-unes de ces questions:

- Comment les succès technologiques actuels de l'IA, par des techniques d'apprentissage, peuvent-ils contribuer à «faire comprendre » la nature profonde et la véritable complexité des traitements qu'exige l'activité de langage?

- Quelles sont les propriétés observables qui permettent de distinguer la place singulière des langues parmi les systèmes sémiotiques observés, par exemple, dans la communication animale?

- Quels sont les processus mis en place par la traduction humaine qui aboutit à des variations souvent pertinentes et se prêtant à des discussions fructueuses à propos de textes traduits 58 ?

Remarque: TraduXio est un environnement numérique pour la traduction collaborative et multilingue de textes culturels. La traduction ne consiste pas en un transfert entre deux langues (source/cible) mais en un passage d'un texte singulier vers d'autres textes singuliers,

\footnotetext{
57 Y. Le Cun, Quand la machine apprend. La révolution des neurones artificiels et de l'apprentissage profond. Paris, Odile Jacob, 2019.

58 Voir, par exemple, Ph. Lacour et Y. Goncharova "Traduxio: nouvelle expérience en traduction littéraire », Traduire, Société Française des Traducteurs, numéro 225, automne 2011.
} 
dans plusieurs langues. La concordance multilingue permet de comparer les différentes versions d'un même texte, stimulant la créativité des traducteurs: des filtres permettent de rechercher des suggestions de façon pertinente, en fonction de catégories introduites par les utilisateurs pour exploiter les corpus de façon intelligente. Cet environnement numérique est certainement un outil qui peut nous aider à étudier les mécanismes de traduction.

- Quels sont les mécanismes cognitifs utilisés mis en œuvre par les humains lorsqu'ils synthétisent plusieurs textes, quelquefois de domaines assez différents, en dégageant certaines prises de position de leurs auteurs, par exemple en identifiant les évaluations souvent associés aux citations empruntées à d'autres auteurs?

- Lorsque l'enfant acquiert son activité langagière, a-t-il recours seulement à un apprentissage qui reposerait uniquement sur les données fournies par son entourage adulte?

- S'il est certain que l'environnement d'une langue maternelle est nécessaire à l'acquisition et à la maîtrise d'une langue, quelle est la part de l'innéisme par rapport aux constructions d'inférences, certaines à la suite d'une abstraction adductive (au sens de C.S. Peirce)?
Tous ces sujets restent plus que jamais d'actualité. Il ne nous paraît pourtant pas évident que les seules techniques d'apprentissage, même profond, qui nécessitent des entrainements longs et avec une masse très importante de données, et sans faire appel à des représentations sémantiques et métalinguistiques, soient des voies totalement adéquates pour arriver à donner des réponses pertinentes aux questions précédentes. Après les résultats technologiques spectaculaires de l'IA actuelle dans certains domaines du traitement des langues, l'IA future ne devra-t-elle pas prendre à nouveau en compte la construction et la prise en compte des significations, avec des changements de leurs représentations, pour mieux simuler l'activité de langage et pour mieux identifier et comprendre les capacités cognitives spécifiques de l'homme? La question reste ouverte à notre époque où l'IA contemporaine réduit presque tous ses traitements aux seules techniques par apprentissage, en faisant parfois alliance avec les neurosciences, pour sembler vouloir apporter des réponses satisfaisantes à tous les problèmes liés à la sémantique et aux problèmes de catégorisation étudiés de plus en plus profondément 59 , Ne conviendrait-il pas de réfléchir sur les conditions épistémologiques d'une véritable interdis-

\footnotetext{
${ }^{59}$ Nous étudions avec A. Pascu ces problèmes dans le cadre de la Logique de la Détermination des objets (DO), voir les notes précédentes.
} 
ciplinarité, en visant, par exemple, à élargir l'horizon actuel de la logique moderne et en établissant des rapports plus pertinents entre les neurosciences et les sciences humaines, dont la linguistique, en tant compte des problèmes posés par ces deux groupes de disciplines scientifiques?

\section{Quelques remarques pour conclure}

Revenons sur quelques distinctions théoriques qui ont été introduites au cours de cet article et qui réclament quelques explications supplémentaires.

La première distinction concerne la pragmatique interne $v s$ pragmatique externe. La pragmatique concerne l'étude des rapports entre, d'un côté, un système sémiotique, appréhendé par sa syntaxe et sa sémantique et, d'un autre côté, les utilisateurs de ce système. Les langues occupent une place particulière parmi les systèmes sémiotiques puisque chaque langue donne une place signifiante directement observable aux diverses relations qu'entretiennent, au cours d'un discours (et pas uniquement dans un dialogue), l'énonciateur avec son coénonciateur qui sont des utilisateurs de la langue. Les distinctions énonciateur vs locuteur et coénonciateur $v s$ allocutaire sont étroitement liées à la distinction entre les deux pragma- tiques. Beaucoup de textes donnent souvent peu d'information sur le locuteur qui écrit et sur celui qui parle dans un texte, d'où les divers sens que l'on peut parfois attribuer à un même texte.

Remarque: La texte de la pièce de théâtre La nuit juste avant les forêts de Bernard-Marie Koltès, comprend 60 pages sans aucun point, mais il est très ponctué par ailleurs. Le spectateur, qui est placé devant une seule énonciation d'un seul énonciateur qui s'adresse à plusieurs coénonciateurs (absents) qui parfois lui répondent ou semblent avoir répondu. est ainsi amené à se poser les questions: Quel est le locuteur qui est représenté par l'énonciateuracteur? Où parle-t-il? A qui s'adresse-t-il? A lui-même ou à une personne déterminée mais absente? Pourquoi parle-t-il? Quel est le sens de cette énonciation qui semble ne jamais finir? L'analyse énonciative d'un tel texte ne devient possible que si l'on se réfère à un énonciateur dans le cadre d'une pragmatique interne en le distinguant du locuteur. On peut montrer, par ailleurs, que la structuration textuelle présente des analogies avec certaines fugues musicales $\sqrt[60]{60}$

A côté des relations déictiques (décrites usuellement comme des opérations d'embrayage), de nombreuses formes temporelles, aspectuelles et modales, grammaticalisées dans certaines langues, sont les traces d'une relation entre l'énonciateur et un contenu prédicatif (un dictum); ces traces indiquent, par le biais de signes linguistiques précis, «l'attitude» adoptée vis-à-vis de ce contenu. Cette attitude, ou prise en charge énoncia-

\footnotetext{
${ }^{60}$ Voir I. Merkoulova, C. Desclés et J.-P. Desclés, «L'inscription fuguée dans le texte de Bernard-Marie Kolès La nuit juste avant les forêts ", Exposé au Séminaire de sémiotique, Université de Paris-Sorbonne, 1/04/.2006; il a été repris et publié dans Les sciences cognitives: questions et perspectives. Actes du séminaire franco-russe, Moscou, 21-22 septembre 2010, pp. 120-149.
} 
tive, n'est évidemment pas, comme nous l'avons déjà remarqué, une relation entre l'énonciateur et son énoncé, puisque c'est l'énoncé qui exprime le résultat de cette prise en charge par l'énonciateur. L'énonciateur est indéterminé, c'est une sorte de variable énonciative; cette manifestation énonciative de l'énonciateur et de tous ses corrélats, relève de la pragmatique interne de la Langue (au sens de Saussure). Quant au locuteur, avec toutes ses déterminations référentielles, il est une entité externe au système de la Langue; il instancie l'énonciateur dans le référentiel externe; il est parfois possible de préciser certaines déterminations référentielles du locuteur et les conditions externes de sa locution, soit en les insérant dans le discours, soit en allant au-delà de ce qui est inscrit dans le discours (ou le texte), en convoquant alors des connaissances externes supplémentaires qui viennent s'ajouter à la signification qui se dégage du seul discours (ou texte). Toutes les connaissances externes au processus de construction de la signification d'un discours relèvent d'une pragmatique externe. Les manifestations linguistiques de l'énonciateur-utilisateur s'étendent au coénonciateur qui est, lui aussi, un utilisateur du système sémiotique de la Langue. Toutes ces relations relèvent de la pragmatique interne des langues. Les descriptions mé- talinguistiques des langues, construites par le linguiste, doivent représenter les opérations qui relèvent de la pragmatique interne. Les opérations énonciatives qui indiquent des ruptures (avec d'éventuelles synchronisations locales) entre référentiels, contribuent à rendre évident le besoin de distinguer deux pragmatiques. Les approches sémiotiques qui ne font pas une telle distinction, qualifient de pragmatique ce qui est pour nous une pragmatique externe; ainsi, la théorie des actes de langage de J. Searle et D. Vanderveken 6 , qui, sur plusieurs points théoriques et descriptifs, rejoint des conceptualisations élaborées dans la théorie de l'énonciation, ne prend pas en compte cette différence entre pragmatiques, puisqu'elle opère uniquement avec des situations dénotatives localisées dans le référentiel externe, ce qui la conduit à assimiler l'énonciateur au locuteur et le coénonciateur à l'allocutaire.

La plupart des systèmes sémiotiques, autres que les langues, ne possèdent pas une pragmatique interne; cette dernière donne à un utilisateur la capacité de reproduire, plus ou moins directement, les paroles d'autrui ou de transmettre par un acte langagier des situations complètement imaginaires et/ou construites par abstraction et localisées dans un référentiel distinct du monde de la perception directe ou indi-

\footnotetext{
61 Voir J. Searle et D. Vanderveken, Foundations of Illocutionary Logic, Cambridge, Cambridge University Press, 1985; C. Jaci de Souza Melo et D. Vanderveken, "Les universaux logiques et linguistiques relatifs aux forces illocutoires », in J.-P. Desclés et F. Le Priol, Annotations automatiques et recherche d'information, Paris, Hermès, Lavoisier, 2009, pp. 303-328.
} 
recte. Les animaux possèdent des systèmes assez complexes de communication (systèmes gestuels observés chez les grands singes, ou système de danses des abeilles), mais ils ne possèdent pas, semble-t-il, des systèmes de repérages ego-centrés et des moyens de d'énoncer des discours rapportés attribués à un tiers; les systèmes de communication des animaux sont plutôt exclusivement géo-centrés et ne sont pas compatibles avec une pragmatique interne. 62

La seconde distinction oppose signification et sens. Dans la littérature linguistique, ces deux mots sont souvent employés l'un pour l'autre. Pourtant, sens connote une orientation vers une cible, ce qui n'est pas le cas de signification, beaucoup plus neutre sur ce point. La détermination du sens d'une expression, d'un énoncé, d'un discours s'appuie en général sur une construction préalable de sa signification, et lui ajoute éventuellement des informations qui contribuent à indiquer une direction vers une "bonne compréhension » en évitant d'éventuels malentendus. Ces informations sont souvent empruntées à une pragmatique externe qui introduit des connaissances sur les relations entre un locuteur et ceux à qui est destinée le discours; ces informations peuvent être, par exemple, des références implicites à d'autres discours qui l'ont précédé ou qui le suivront ${ }^{63}$ Les textes littéraires, juridiques, philosophiques, religieux, parfois même des textes scientifiques, possèdent une signification qu'une analyse linguistique rigoureuse peut reconstruire et expliciter, en en faisant apparaître d'éventuelles ambiguïtés, mais le sens de ces textes est souvent renouvelé par des «lectures » qui, introduisent de "nouvelles » connaissances et parfois un autre "point de vue », en proposant une nouvelle perspective interprétative, c'est-à-dire un certain sens que la signification seule ne révélait pas. A titre d'exemples, outre les commentaires et interprétations des textes de la Bible ou du Coran, on peut citer les différentes commentaires et interprétations des textes philosophiques, comme ceux de Platon, d'Aristote, de Rousseau, de Heidegger...

La distinction signification vs sens, s'oppose évidemment à la dénotation d'une expression. Avec G. Frege, on a appris que les significations (comme celles l'étoile du soir et l'étoile du matin) ne sont évidemment pas réductibles à leurs dénotations. Dans notre approche énonciative, la dénotation est relative à un référentiel, elle n'est donc pas absolue et ne se réduit pas à une référence dans le référentiel externe, indépendant des conditions d'énonciation. Dire que

\footnotetext{
62 Voir, par exemple, l'analyse linguistique par E. Benveniste du système de communication des abeilles, effectué à partir des observations de K. von Frisch in E. Benveniste, Problèmes de linguistique générale, Gallimard, Paris, 1965.

63 C'est, par exemple, le cas de nombreux textes de la Bible qui se réfèrent souvent indirectement à d'autres textes, comme nous l'avons montré dans notre analyse énonciative du Livre de Jonas dans J. P. Desclés et G. Guibert, 2011, o.c. pp. 125-209,
} 
« le sens est son usage » comme le dit L. Wittgenstein est insuffisant. En effet, la détermination du sens d'une expression suppose préalablement la construction de sa signification qui n'est pas réductible à la seule compréhension de ses composants et de leurs usages. Mais cela nous nécessiterait de longues discussions.

La troisième distinction entre opération et opérateur n'a pas été spécialement définie dans cet article. Elle est cependant pour nous fondamentale et elle a fait l'objet de plusieurs publications 64. Pour définir une opération, il est nécessaire de préciser d'un côté, l'ensemble des arguments de l'opération à effectuer et, d'un autre côté, l'ensemble des résultats de l'opération quand elle sera ou a été effectuée. L'opérateur est plus abstrait que l'opération; sa caractérisation est précisée par ses compositions avec d'autres opérateurs et par ses possibilités de transformation au moyen d'opérateurs abstraits, par exemple, les combinateurs de la logique combinatoire (LC). Comme nous l'avons déjà dit, la LC est une logique de compositions et de transformations intrinsèques d'opérateurs quelconques, indépendamment des domaines et propriétés de ces domaines sur lesquels opèrent les opérateurs composés ou transformés. Par-là, la LC rejoint le niveau d'abstraction visé en mathéma- tiques par la théorie des catégories de S. Eilenberg, S. Mac Lane et F.W. Lawvere, lorsqu'un opérateur est pensé comme flèche composable. Il nous apparaît alors que le concept d'opérateur n'est sans doute guère éloigné de la notion du 'métaconcept d'opération' de G.-G. Granger:

"Il semble donc que le point de vue de la théorie des Catégories privilégie l'aspect opératoire de l'univers mathématique (alors que le point de vue ensembliste privilégie plutôt l'aspect objectal). (...) A ce niveau d'abstraction, qui est celui des mathématiques, c'est principalement le système opératoire qui est formulé par les axiomatiques; les objets $y$ apparaissent seulement, pour ainsi dire, comme des concrétions possibles aux nœuds du réseau dessiné par les opérations. »

(G.-G. Granger, formes opérations objets, Paris, Vrin, 1994, p. 388).

Dans les langues naturelles, les unités grammaticales, ou grammèmes, jouent manifestement un rôle opératoire en prenant justement une forme d'opérateurs qui se composent avec d'autres opérateurs (des grammèmes et

${ }^{64}$ Voir J.-P. Desclés et al. vol. II, 2016, pp. 123-148, pp. 563-602. 
des lexèmes) ou qui transforment des opérateurs (par exemple des lexèmes) indépendamment des objets concrets représentés. Il y a là tout un domaine à explorer.

Notre approche du langage et des langues est-elle compositionnelle? La notion de compositionnalité, très utilisée en sémantique formelle, reste assez ambiguë. En effet, elle peut être simplement réduite à vouloir dire que la signification d'une expression serait la 'somme' ou encore serait 'fonction' des significations de ses composants. Dans une approche plus technique, le terme vague de 'fonction' peut être pris au sens précis des mathématiques, mais il convient alors de décrire comment construire exactement cette fonction. Notre approche de la sémantique (grammaticale et lexicale), simplement esquissée dans cet article, pourrait laisser penser que nous nous situons dans une sémantique formelle où l'analyse sémantique reviendrait à décrire la fonction qui associe une interprétation à chaque unité de la structuration syntaxique, selon une correspondance fonctionnelle. Or, notre approche est plus complexe. En effet, en utilisant le formalisme de la LC typée de Curry, qui accueille les représentations linguistiques et métalinguistiques sous la forme d'expressions applicatives, les combinateurs autorisent des constructions beaucoup plus complexes que les simples compositions fonctionnelles de fonctions ensemblistes, comme le montrent des exemples de construction de significations que nous avons traités. 65 Si notre approche de la sémantique peut être qualifiée de compositionnelle, c'est en lui donnant le sens technique de composition (et de transformation) d'opérateurs par des combinateurs de la LC ou par des processus opératoires équivalents formulés dans le cadre de la théorie des catégories.66

Les exemples détaillés traités à l'aide de la LC dans nos publications sont des énoncés. Pour dépasser cette seule dimension discursive, il faut recourir à des 'marqueurs discursifs' utilisés pour structurer et organiser une succession d'énoncés sous la forme de discours et de textes cohérents. L'identification et le traitement formel de ces différents marqueurs discursifs commencent à être cernés et étudiés par la linguistique actuelle ${ }^{67}$. Il faut cependant

\footnotetext{
65 Voir Desclés et al., volume II, 2016, o.c.. Si le combinateur B de la LC exprime la composition fonctionnelle de fonctions de la théorie des ensembles, d'autres combinateurs effectuent des compositions beaucoup plus complexes, par exemple, des compositions en parallèle d'opérateurs intriqués appliqués aux mêmes opérandes, ou encore des duplications d'opérandes d'un même opérateur binaire, ou encore des construction d'opérateurs à partir d'une expression applicative donnée, voir Desclés et al. vol. I, 2016, o.c. , pp. 41-79.

66 Voir J.-P. Desclés, Opérateurs, opérations : méthodes intrinsèques en informatique fondamentale, thèse d'état en mathématiques, publiée dans la collection ERA 642, Université de Paris 7, 1981. Il existe des liens mathématiques pertinents entre la théorie des catégories et la LC typée.

67 Citons comme exemples de marqueurs discursifs, ceux qui sont analysés dans C. Rossari, Connecteurs et relations de discours: des liens entre cognition et signification, Nancy, Presses Universitaires de Nancy, 2000.
} 
gérer la polysémie de ces marqueurs discursifs en tenant compte de certains indices linguistiques présents dans le contexte de ces marqueurs avec la méthode d'exploration contextuelle 68. Par ailleurs, nous avons entrepris de travailler la construction des significations sur des textes entiers.69

Pour entreprendre une analyse approfondie de la signification puis du sens des discours et des textes, il nous semble cependant indispensable de savoir au préalable dégager et con- struire les significations des énoncés qui en sont les composantes, de façon à montrer comment différents marqueurs discursifs organisent, par une autre forme de compositionnalité discursive, les significations de chaque énoncé en une signification structurée plus globale, en sachant que la détermination du sens d'un discours et d'un texte n'est pas réduite à la signification mais qu'il en dépend. C'est encore tout un programme de recherche.

\section{Références}

BALLY, Ch. Linguistique générale et linguistique française, Berne, Editions Francke, 1934/1965;

BARBUT, M. «Topologie générale et algèbre de Kuratowski », Mathématiques et sciences humaines, 1965, pp. 11-27.

BENVENISTE, E. Problèmes de linguistique générale, Paris, Gallimard, 1965, 1974.

BERRY, G. L'Hyperpuissance de l'informatique. Algorithmes, données, machines, réseaux, Odile Jacob, Paris, 2017 pp. 448451.

BISKRI, I. et DESCLES, J-P. « Applicative and Combinatory Categorial Grammar (from syntax to functional semantics) », in R. MITKOV et N. NIKOLOV (eds), Recent Advances in Natural Language Processing, John Benjamins, 1997, pp. 71-84.

BLAIS, A. et DESCLÉS, J.-P. « L'annotation discursive de textes et son application au résumé automatique », in Y. Jeanneret et N. Meeùs (dir .) Que faisons-nous du texte? Presses de l'Université de Paris Sorbonne, Paris, 2012, pp. 47-75; J. P. Desclés et G. Guibert 2011, o.c..

BOGACKI, K. et al., Dictionnaire sémantique et syntaxique des verbes français, Warszawa, Panstwowe Wydawnictwo Naukowe, 1983.

BUBER, M. Je et Tu, [Ich und Du, 1923], Paris, Aubier-Montaigne, 1969.

COLAS-BLAISE, M.; PERRIN, L. et TORE, G. M. L'énonciation aujourd'hui, un concept clé des sciences du langage, Paris, Lambert-Lucas, 2016.

CULIOLI, A. et DESCLÉS, J.-P., avec la collaboration de R. KABORE et Dj. KOULOUGHLI. Systèmes de représentations linguistiques et métalinguistiques. Les catégories grammaticales et le problème de langues peu étudiées, Université Paris 7, 1981, 141 pages, publié dans la Collection ERA 642.

CUliOLI, A. Pour une linguistique de l'énonciation, Tomes 1,2,3, Paris, Ophrys, 1990, 1999.

CULIOLI, A. Pour une théorie de l'énonciation, formalisme et opération de repérage, Tome 2, Paris, Ophrys, 1999, p. 25.

CULIOLI, A. Variations sur la linguistique, Entretiens avec Frédéric Fau, Paris, Klincksieck, 2002, p. 28.

CURRY, H. B. and FEYS, R. Combinatory Logic, Vol. I, 1958; J. R. Hindl ey and J. P. Seldin, Lamda-calculus and Combinators, an introduction, Cambridge: Cambridge University Press, 2008.

\footnotetext{
${ }^{68}$ Voir, entre autres publications, J.P. Desclés et F. Le Priol, Annotations automatiques et recherche d'information, Paris, Hermès, Lavoisier, 2009.

${ }^{69}$ Voir J.-P. Desclés, « Analyse syntaxique et analyse discursive », in Dan Van Raemdonck (dir.) Modèles syntaxiques. La syntaxe à l'aube du XXIe siècle, P.I.E. Peter Lang, Bruxelles, 2008, pp. 165-182; A. Blais et J.-P. Desclés, « L'annotation discursive de textes et son application au résumé automatique », in Y. Jeanneret et N. Meeùs (dir .) Que faisons-nous du texte? Presses de l'Université de Paris Sorbonne, Paris, 2012, pp. 47-75; J. P. Desclés et G. Guibert 2011, o.c..
} 
DESCLÉS, J.-P. “A cognitive and conceptual approach to tenses and aspect markers”. in GUENTCHÉVA, Z. (ed.) Aspectuality and Temporality. Descriptive and theoretical issues, Amsterdam / Philadelphia, John Benjamins, 2016, pp. 27-60.

DESCLÉS, J.-P. “Brève généalogie des grammaires catégorielles”, Verbum, tome XL, N², 2018, pp. 143-172 ; J.P. Desclés et al., Logique combinatoire et -calcul, Vol. I, Toulouse, Cépaduès, 2016, pp. 201-234; voir aussi Vol. II, 2016, o.c., pp. 304-355.

DESCLÉS, J.-P. « Les représentations cognitives du langage sont-elles universelles? », in Essais sur le langage, logique et sens commun, Fribourg, Suisse, Editions Universitaires, 1998, pp.53-81.

DESCLÉS, J.-P. "Analyse syntaxique et analyse discursive ", in Dan Van Raemdonck (dir.) Modèles syntaxiques. La syntaxe à l'aube du XXIe siècle, P.I.E. Peter Lang, Bruxelles, 2008, pp. 165-182.

DESCLÉS, J.-P. «Cognition, compilation et langage », in Chazal et Terasse (eds), Philosophie du langage et informatique, Paris, Hermès, 1996, pp. 103-145

DESCLÉS, J.-P. « Le problème de la polysémie verbale: donner en français », Cahiers de lexicologie, 2011-1, n98, pp. 95-111.

DESCLÉS, J.-P. « Les mathématiques de la grammaire d'opérateurs de Zellig Harris », in C. Martinot et al., Perspectives harissiennes, Cellule de recherche linguistique, Paris, 2016, pp. 83-105.

DESCLÉS, J.-P. «Opérations et opérateurs énonciatifs », in COLAS-BLAISE, M.; PERRIN, L. et TORE, G. M. L'énonciation aujourd'hui, un concept clé des sciences du langage, Paris, Lambert-Lucas, 2016, pp. 69-88.

DESCLÉS, J.-P. «Quelques opérations énonciatives » in J. DAVID et R. MARTIN, Logique et niveaux d'analyse linguistique, Paris, Klincksieck, 1976.

DESCLÉS, J.-P. « Représentation des connaissances: archétypes cognitifs, schèmes conceptuels, schémas grammaticaux ", Actes sémiotiques, Documents VII, № 69-70, EHESS-CNRS, 1985, pp. 5-51; voir aussi Desclés et al., volume II, 2016, o.c., pp. 451-496.

DESCLÉS, J.-P. et GUENTCHÉVA, Z. "Universals and Typology”, in R. Binnick (ed), Oxford Handbook of Tense and Aspect, New York, Oxford University Press, 2012, pp. 123-154.

DESCLÉS, J.-P. et GUENTCHÉVA, Z. "Référentiels aspectuo-temporels: une approche formelle et cognitive appliqué au français », Bulletin de la Société de Linguistique de Paris, 56 (1), 2012, pp. 95-127.

DESCLÉS, J.-P. et GUENTCHÉVA, Z. "Quasi topological representations (QTR) of spatial places and spatio-temporal movements in natural languages », in G. MAROTTA et al., Space in Language, Proceedings of the Pisa International Conference, Edizioni ETS, Firenze, 2010, pp. 213-233.

DESCLÉS, J.-P. et GUIBERT, G. Le dialogue, fonction première du langage, Analyse énonciative de textes, Paris, Honoré Champion, 2011, pp. 49-73.

DESCLÉS, J.-P. et LE PRIOL, F. Annotations automatiques et recherche d'information, Paris, Hermès, Lavoisier, 2009.

DESCLÉS, J.-P. et PASCU, A. “Logic of Determination of Objects: How to articulate 'Extension' with 'Intension' and 'Objects' with 'concepts' ", Logica Universalis 5/1, 2011, pp. 75-89.

DESCLÉS, J.-P. Opérateurs, opérations: méthodes intrinsèques en informatique fondamentale, thèse d'état en mathématiques, publiée dans la collection ERA 642, Université de Paris 7, 1981.

DESCLÉS, J-P.; GUIBERT, G. et SAUZAY, B. Calculs des significations par une logique d'opérateurs * Vers une logique d'opérateurs; ${ }^{* *}$ Concepts et schèmes analysés par la logique combinatoire, Vol. II, Toulouse, Cépaduès, 2016, pp. 418-493.

DESCLÉS, J-P.; PASCU,A. C.. "Logic of Typical and Atypical Instances of a Concept. |A Mathematical Model”. Axioms, 8(3), 2019, p. 104 et sq.

GRANGER,G.-G. Formes, Opérations, Objets, Paris, Vrin, 1994.

GRIZE, J.-B. Logique moderne III, Paris, Gauthier-Villars, 1973, pp. 37-38.

JACI DE SOUZA MELO, C. et VANDERVEKEN, D. « Les universaux logiques et linguistiques relatifs aux forces illocutoires ", in DESCLÉS, J.-P. et LE PRIOL, F. Annotations automatiques et recherche d'information, Paris, Hermès, Lavoisier, 2009, pp. 303-328.

LACOUR, Ph. et GONCHAROVA, Y. «Traduxio: nouvelle expérience en traduction littéraire », Traduire, Société Française des Traducteurs, numéro 225, automne 2011.

LAWVERE, F. W. et SCHANUEL, S. H. Conceptual Mathematics. A first introduction to categories, Cambridge Univerity Press, 2009.

LE CUN, Y. Quand la machine apprend. La révolution des neurones artificiels et de l'apprentissage profond. Paris , Odile Jacob, 2019.

LÜDI, G. et ZUBER, Cl. (eds), Linguistique et modèles cognitifs. Contributions à l'Ecole d'été de la Société Suisse de Linguistique, ARBA 3, Acta Romanica Basiliensia. XXX, Université de Bâle et Institut Universitaire Kurt Bösch, 1995.

MARR, D. Vision, Freeman and Company, New York, 1982. 
MERKOULOVA, I.; DESCLES, C. et DESCLÉS, J.-P. " L'inscription fuguée dans le texte de Bernard-Marie Kolès $L a$ nuit juste avant les forêts ", Exposé au Séminaire de sémiotique, Université de Paris-Sorbonne, 1/04/.2006; il a été repris et publié dans Les sciences cognitives: questions et perspectives. Actes du séminaire franco-russe, Moscou, 21-22 septembre 2010, pp. 120-149.

PASCU, A. C.; DESCLÉS, J.-P.; BISKRI, I. "A topological approach for the notion of Quasi-topology structure”. South American Journal of Logic, Vol. X, n. X, 2019, pp. 1-18.

PITRAT, J. Métaconnaissances, futur de l'intelligence artificielle, Paris, Hermès, 1990.

RABATEL, Alain. La construction textuelle du point de vue, Paris, Delachaux et Niestlé, 1998.

ROSSARI, C. Connecteurs et relations de discours: des liens entre cognition et signification, Nancy, Presses Universitaires de Nancy, 2000.

SABAH, G. L'intelligence artificielle et le langage, processus de compréhension, Paris, Herrmès, 1989.

SEARLE, J. et VANDERVEKEN, D. Foundations of Illocutionary Logic, Cambridge, Cambridge University Press, 1985.

SHAUMYAN, S.K. A Semiotic Theory of Language, Bloomington, Indiana University Press, 1987.

TESNIERE, L. Éléments de syntaxe structurale, Paris, Klincksieck, 1959/ 1988.

THOM, R. Stabilité structurelle et morphogénèse, John Benjamins, 1973.

VENDLER, Z. "Verbs and times", Philosophical Review, 66, 1957, pp. 143-160.

WARUSFEL, A. Les mathématiques modernes, Paris, Le rayon de la science, 1969, pp. 166-170.

WHITEHEAD, A. Process and Reality, New York, Macmillan, 1929.

WIERZBICKA, A. «La quête des primitifs sémantiques ». Langue française, nº98, Les primitifs sémantiques, 1993, pp. 9-23.

WILDGEN, W. Catastrophe theoretic semantics. An application and elaboration of René Thom'theory, Amsterdam, John Benjamins. 1982.

WINOGRAD, Th. et FLORES, F. L'intelligence artificielle en question, Paris, Presses Universitaires de France, 1986.

Reçu / Recebido: 18/04/2020

Approuvé / Aprovado: 20/04/2020

Publié / Publicado: 20/09/2020 
